\title{
ANALYSIS OF DIRECT THREE-DIMENSIONAL PARABOLIC PANEL METHODS*
}

\author{
PHILIPPE PONCET ${ }^{\dagger}$
}

\begin{abstract}
Adherence boundary conditions for time dependent partial differential equations, via Chorin algorithm, can be reduced to a parabolic problem with Robin-Fourier boundary conditions in the three-dimensional context. In the spirit of panel methods, one establishes an integral formulation whose key point is the estimation of the potential density, introducing a kind of panel method for tangential kinematic boundary conditions. This paper discusses explicit estimations of this density in the general case of an arbitrarily shaped three-dimensional body, which leads to a fast numerical scheme. An error analysis is also provided, involving body smoothness, the Hölder exponent of the density, and whether the body presents torsion or not.
\end{abstract}

Key words. numerical analysis, boundary value problems, integral representation, fundamental solutions, parabolic equations, growth of solutions

AMS subject classification. 65M, 35K20, 65L10, 35B05, 35C15

DOI. $10.1137 / 050625849$

1. Introduction. Numerical techniques aimed at solving partial differential equations involving kinematic boundary conditions, such as the Navier-Stokes equations, have been viewed from many perspectives. These kinematic boundary conditions usually rely on zero velocity field on bodies when considering viscous flows. Despite the fact that these conditions are all mathematically of homogeneous Dirichlet type, fixing velocity value at boundaries, such vectorial boundary conditions have very different meanings physically, depending on the velocity component: while the zero normal component of velocity field on a body is linked to a no-slip-through property, or impermeability, the zero tangential components come from an adherence property, or no-slip condition, not required for ideal fluids relevant to the Euler equations.

The present article focuses on the integral formulation of adherence properties, which is related to a parabolic problem via the Chorin algorithm (instead of elliptic for classical panel methods). We present for the first time the numerical analysis of an ad hoc density evaluation, commonly known as the fastest way to ensure adherence properties.

It is now generally recognized that integral methods provide powerful tools to enforce numerically such boundary conditions. Concerning the normal conditions of velocity, the most common discrete integral technique, known as the "panel method," was pioneered by Hess $[18,19]$ in the 1970s and consists in using a formulation close to electromagnetism [26], that is, in finding a potential of the form

$$
\phi(x)=\int_{\partial \Omega} K(x, y) q(y) \mathrm{d} \sigma(y) .
$$

In this equation (1.1), $K$ is a Green function, whose expression is $(4 \pi|x-y|)^{-1}$ in the full space $\mathbb{R}^{3}$, and $q$ is the density function, defined over domain boundary and

\footnotetext{
*Received by the editors March 3, 2005; accepted for publication (in revised form) January 17, 2007; published electronically October 10, 2007.

http://www.siam.org/journals/sinum/45-6/62584.html

${ }^{\dagger}$ Laboratoire MIP, Department GMM, INSA, 135 avenue de Rangueil, 31077 Toulouse Cedex 4, France (Philippe.Poncet@insa-toulouse.fr).
} 
solution of the following integral equation:

$$
-\frac{q(x)}{2}+\int_{\partial \Omega} n_{x} \cdot \nabla K(x, y) q(y) \mathrm{d} \sigma(y)=g(x),
$$

where $n_{x}$ denotes the normal field to $\partial \Omega$ and $g$ is a given function depending on the problem considered. The velocity is then obtained by differentiation of this potential. Proofs of regularity and well-posedness properties of related discrete operators can be found in the existing literature (see [34], for example). This usually leads to solving a large linear system of the size of the boundary discretization [21], which can be nevertheless efficiently preconditioned [16]. The order of convergence can be under control and possibly high $[3,4,35]$. In order to speed up the computation, a way to proceed is to use multipole methods $[15,33,17]$, which are by definition well adapted for Lagrangian or pointwise formulations [2]. Another way is to provide an estimate of density, which limits convergence order but dramatically decreases computational time since only potential evaluation remains to be computed [13].

The tangential part of kinematic boundary conditions is a completely different matter. In the fluid dynamics context, these conditions are related physically to viscous effects, modeled by the Laplacian operator in the Navier-Stokes equations, which makes them of parabolic type, as opposed to the Euler equations, which are hyperbolic. Since the 1980s, several numerical schemes aimed at splitting apart linearity and nonlinearity have been proposed and implemented in various fields of physics and mathematical physics, in order to use well-fitted numerical techniques taking into account the linearity, or lack thereof.

These splitting techniques, also known as fractional step algorithms, can be basically of first order, or second order when based on the Strang formula, or higher order by using more general Trotter permutation formulae. Splitting the Navier-Stokes equations [22] over a time step leads one to consider successively the Euler equation with only its natural no-slip-though boundary condition, and then a Stokes equation with full no-slip conditions [9]. In its vorticity formulation, the Stokes problem can be reduced to a heat equation, possibly vectorial for three-dimensional configurations, with kinematic boundary conditions [8] relying only on tangential components. From a physical point of view, this heat equation takes into account both near-wall adherence properties and viscous effects in the whole fluid.

Lighthill's model states that these kinematic no-slip conditions for a fluid result from vorticity production on solid boundaries [29]. This production of vorticity has been viewed from many perspectives, involving Dirichlet conditions [7, 37] or Neumann conditions [23, 24], usually constrained by Kelvin's theorem to satisfy conservation of circulation. It has been shown that Neumann conditions are well adapted for nonstationary flows for two-dimensional problems $[8,25]$ or three-dimensional problems in the half-space [9], i.e., without curvature. It has been recently put forward that threedimensional vortical boundary conditions involve the Robin-Fourier condition [11].

By using linearity of the heat equation, it can be split without any approximation into an equation with a generally nonzero initial condition and homogeneous boundary conditions, and another equation with zero initial condition and Robin-Fourier boundary conditions, which can be written as

$$
\left\{\begin{array}{l}
\left.\frac{\partial \omega}{\partial t}-\nu \Delta \omega=0 \text { in } \Omega \times\right] 0, T[ \\
\omega(x, 0)=0 \text { on } \Omega, \\
\left.\nu \mathcal{L}_{x} \omega(x, t)=F(x, t) \text { on } \partial \Omega \times\right] 0, T[
\end{array}\right.
$$


where $\Omega$ is an open set of $\mathbb{R}^{3}, F$ is a boundary source, $\nu$ is the diffusion coefficient, and $\mathcal{L}$, the differential operator defining Robin-Fourier boundary conditions, can be written as

$$
\mathcal{L}_{x}=\beta(x) \operatorname{Id}+n(x) \cdot \nabla,
$$

where $n(x)$ denotes the inward normal field to $\partial \Omega$ and $\beta$ denotes a continuous and bounded function from $\partial \Omega$ to $\mathbb{R}$, bounded by $\beta_{0}$ (i.e., $|\beta(x)|<\beta_{0}$ for all $x \in \partial \Omega$ ). Throughout the present paper, $\Omega$ is supposed to be an open set such that $\partial \Omega$ is a two-dimensional submanifold of $\mathbb{R}^{3}$, of class $\mathcal{C}^{2+\lambda}$, and the source $F$ is supposed to be a bounded and continuous function on $\partial \Omega \times[0, T]$. The solution of this heat equation can be found in its integral formulation:

$$
\omega(x, t)=\int_{0}^{t} \int_{\partial \Omega} G_{\xi, \tau}(x, t) \widetilde{\mu}(\xi, \tau) \mathrm{d} \sigma(\xi) \mathrm{d} \tau,
$$

where $\sigma$ is a measure on $\partial \Omega$ induced by the Lebesgue measure and $G_{\xi, \tau}$ is the parametrix [20,27], which is, in the case of an isotropic heat equation, simply the following three-dimensional Gaussian function:

$$
G_{\xi, \tau}(x, t)=\widehat{G}(x-\xi, \nu(t-\tau)) \text { with } \widehat{G}(x, \eta)=\frac{e^{-x^{2} / 4 \eta}}{(4 \pi \eta)^{3 / 2}},
$$

whose standard deviation is $\sqrt{2 \nu(t-\tau)}$. The density field $\widetilde{\mu}$ defined on $\partial \Omega$ is the solution of the following Volterra-type integral equation:

$$
-\frac{1}{2} \widetilde{\mu}(x, t)+\nu \int_{0}^{t} \int_{\partial \Omega} \mathcal{L}_{x} G_{\xi, \tau}(x, t) \widetilde{\mu}(\xi, \tau) \mathrm{d} \sigma(\xi) \mathrm{d} \tau=F(x, t),
$$

which admits a unique continuous and bounded solution over $\partial \Omega \times[0, T]$ under some minimalistic hypothesis of smoothness, discussed in [14]. Existence, uniqueness, and regularity of solutions of the heat equation and this integral equation have been intensively treated in the literature, many results being summarized in [14] and [28].

Joint equations (1.5)-(1.7) are similar in spirit to (1.1)-(1.2), with both providing a potential aimed at satisfying boundary conditions, and could be named "parabolic panel method." Nevertheless, such a panel method involves fully time dependent densities, which is a function of two variables (instead of one in (1.5)). Moreover, the integrodifferential operator in (1.7) is twice integrated, in time and space. These two remarks make joint equations (1.5)-(1.7) much more difficult to handle than classical panel methods and lead to a much higher degree of computational complexity.

Fast algorithms estimating density $\widetilde{\mu}$ are consequently of fundamental interest in order to make the integral method usable in practice, especially in a three-dimensional context. A way to obtain a fast algorithm is to estimate analytically the density $\widetilde{\mu}$ as a function of the source $F$, viscosity $\nu$, time $t$, the coefficients of operator $\mathcal{L}$, and local invariants of $\partial \Omega$ such as its curvature.

Carrying out density estimation from (1.7) has been performed for two-dimensional bodies [24] and for the three-dimensional case of the half-plane [9] (which comes directly via a tensorialization for the two-dimensional case). Nevertheless, the existing literature either considers pure Neumann boundary conditions or simply neglects curvature effects, sometimes involving some hypothesis on nondependency on time (usually not mathematically valid) and in any case not followed by any mathematical analysis on the order of the method. 
The present paper provides such an analysis for the more general problem of Robin-Fourier boundary conditions and a class of noncompact domains, in establishing, proving, and illustrating that the early behavior of the density can be explicitly given by the following formula:

$$
\widetilde{\mu}(x, t)=\frac{-2 F(x, t)}{1+2(\bar{\kappa}(x)-\beta(x)) \sqrt{\nu t / \pi}}+\mathcal{O}\left(t^{\gamma}\right),
$$

where $\bar{\kappa}(x)$ is the mean curvature of $\partial \Omega$ in $x$, and where $\gamma$ can reach different values among $] 1 / 2,3 / 2]$ in the present study, depending on regularity of $\partial \Omega$ and whether or not $\partial \Omega$ presents torsion. This result can then be used directly as a numerical scheme in formula (1.5), its order being led by the value of $\gamma$.

One can notice that Neumann boundary conditions can lead to qualitatively good results at high Reynolds numbers since the relative curvature $\bar{\kappa} \sqrt{\nu}$ tends toward 0 . Nevertheless, the Dirichlet part of the Robin-Fourier boundary conditions is linked to the boundary curvatures [11], and is of the same order of the mean curvature effect, as shown in formula (1.8) above. Studying the full Robin-Fourier conditions is consequently of fundamental importance for engineering concerns on viscous flows, especially since the research community finds new interests in micro- and nanotechnologies, which involve small scales where viscous effects are potentially dominant. In this context, neglecting curvature can lead to dramatic errors in numerical simulation of fluids, especially when considering nonstationary dynamics whose prediction requires direct numerical simulation. Moreover, even for macroscopic devices, some new generation Lagrangian schemes such as vortex in cell (VIC) (see [10]) or smooth particle hydrodynamics (SPH) (see [6]) are very stable and can be used with large time steps. Consequently, this enlarges numerical viscous scales, which are of order $\sqrt{\nu \delta t}$ (where $\delta t$ is the time step), and makes the curvature effects orders of magnitude stronger than standard numerical methods, such as spectral or finite element schemes, whose time scales are limited due to strong stability conditions related to transport terms.

The outline of the paper is as follows. Section 2 provides various preliminary properties. Section 2.1 gives a few well-known properties of fundamental solutions of the heat equation, results more or less already established in the literature. A few conditions are then set in section 2.2 in order to provide a good environment for differential and integral calculus for the following sections. In section 2.3 , we show that integral calculus restricted to a local area is an accurate approximation of the global calculus at any order of time. This section also extends a classical result on Hölder continuity of the double heat layer to a class of noncompact manifolds. Section 3 provide convergence results and error estimations of geometrical approximation when the integrodifferential operator is computed on the local quadratic osculating manifold instead of the manifold itself. Section 4 shows that the heat layer of unit density on the best quadratic approximation of the surface can be determined at its main order with error estimation. Finally, section 5 presents the achievement of the present work, and Theorem 5.1 shows that the value of the approximated heat layer obtained in section 5 is a valid value at the first order in time. Theorem 5.3 shows that sufficiently smooth torsionless manifolds allow one to reach order $3 / 2$. The link between these results is displayed in Figure 1. Sections 6 and 7 give several examples illustrating some statements of previous sections, in cylindrical and toroidal geometries, respectively. These examples show that estimates given by Theorems 5.1 and 5.3 are optimal and describe their application to kinematic boundary conditions. 


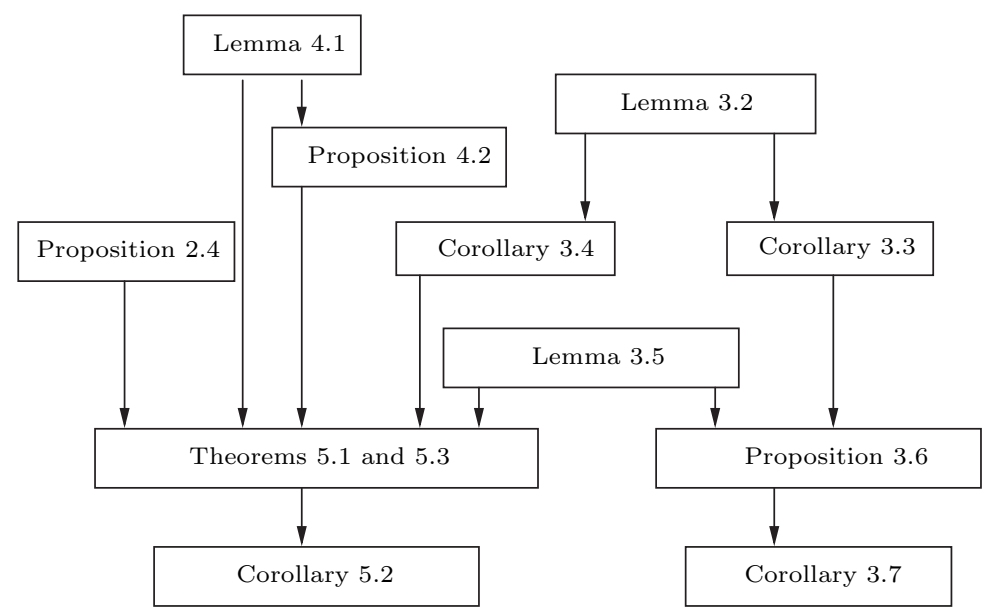

FIG. 1. Relations between results leading to Theorems 5.1 and 5.3 (Lemma 3.1 and Proposition 2.3 are often involved in different proofs).

2. Preliminary work. This section presents well-known results on parabolic problems in section 2.1 and the hypotheses that are required for the present study in section 2.2. Accuracy of domain restriction is then analyzed in section 2.3, with a specific proof valid for noncompact manifolds.

2.1. Well-known results. One first introduces Friedman's notion of Hölder continuity for parabolic problems: a function $\omega$ is said to be $\vartheta$-Hölder continuous over $\Omega \times[0, T]$ if there exist two constants $C$ and $\vartheta$, independent of $x, y, t$, and $s$, such that

$$
|\omega(x, t)-\omega(y, s)| \leqslant C\left(|x-y|^{\vartheta}+|\nu(t-s)|^{\vartheta / 2}\right)
$$

for all $(x, t)$ and $(y, s)$ in $\bar{\Omega} \times[0, T]$, where $|\cdot|$ denotes both the Euclidean norm of $\mathbb{R}^{3}$ and the absolute value, depending on the context.

Throughout the paper, $|\cdot|$ will be used for the Euclidean norm in $\mathbb{R}^{n}$ (absolute value when $n=1$ ), $\|\cdot\|$ for norms of functions, and $\||\cdot \||$ for linear operators (not necessarily Euclidean for double and triple norms, but $\mathbb{L}^{\infty}$ norm most times and $\mathbb{L}^{1}$ occasionally).

The integral operator is of fundamental interest for the present study, since its value at the leading order is the effect of curvature. It is a double layer of density $f$ with respect to the heat kernel and depends on surface location and time (on $\partial \Omega \times[0, T]):$

$$
\widetilde{\mathcal{H}}(x, t) f=\nu \int_{0}^{t} \int_{\partial \Omega} \mathcal{L}_{x} G_{\xi, \tau}(x, t) f(\xi, \tau) \mathrm{d} \sigma(\xi) \mathrm{d} \tau
$$

for any density $f$ bounded and continuous on $\partial \Omega \times[0, T]$.

Furthermore, from [31] and Theorem 4 of Chapter 5 of [14], one gets the following result.

Corollary 2.1. Under the notation above, if $f$ is a continuous and bounded function on $\partial \Omega \times[0, T]$, and $\partial \Omega$ is a compact two-dimensional submanifold of $\mathbb{R}^{3}$ of class $\mathcal{C}^{1+\lambda}$, then $\widetilde{\mathcal{H}}(x, t) f$, as a function of $x$ and $t$, is $\vartheta$-Hölder continuous on $\partial \Omega \times[0, T]$ for any exponent satisfying $\vartheta<2 \min (\lambda, 1) / 3$. 


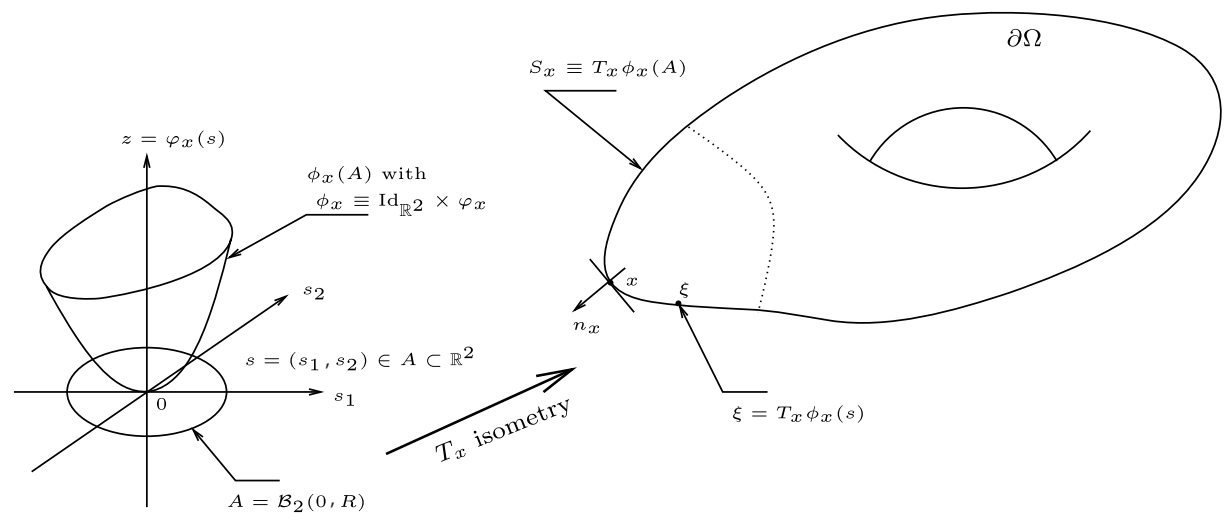

FIG. 2. Geometrical setup and main notation.

Nevertheless, for a manifold of class at least $\mathcal{C}^{2}$, this corollary gives that $\widetilde{\mathcal{H}}(x, t) f$ is $\vartheta$-Hölder continuous for any exponent less than $2 / 3$. In our context, one can go further than this result. Indeed, Corollary 2.1 is independent of the dimension of space and is valid for manifolds presenting less regularity (i.e., manifolds of class $\mathcal{C}^{1+\lambda}$ ) than our present considerations. In the present context, set up in section 2.2, the following proposition (more general than Corollary 2.1) holds.

Proposition 2.2. Let $\widetilde{\mathcal{H}}$ be the integrodifferential operator defined by formula (2.2). Under conditions (C1)-(C5), with $\beta: \partial \Omega \rightarrow \mathbb{R}$ and $f: \partial \Omega \times[0, T] \rightarrow \mathbb{R}$ two bounded and, respectively, $\vartheta_{\beta}$ - and $\vartheta_{f}$-Hölder continuous functions, we have that $\widetilde{\mathcal{H}}(\cdot, \cdot) f$ is $\vartheta^{*}$-Hölder continuous with any $\vartheta^{*}=\min \left(1-\varepsilon, \vartheta_{\beta}, \vartheta_{f}\right)$ for all $\varepsilon>0$.

One can see with this proposition that the maximum Hölder exponent is bounded by the density's, which forbids us from deducting regularity of $\widetilde{\mu}$ from the regularity of $\widetilde{\mathcal{H}}$.

Moreover, Proposition 2.2 shows that the regularity of the density depends on the manifold considered, on the regularity of the source, and on the regularity of the coefficient defining the Dirichlet part of the boundary condition (i.e., the three are involved and arise at the same order in the regularity analysis). The regularity consequently has to be analyzed case by case and will not be discussed in the present paper. To proceed, one can refer to [36] and references therein or $[5,1]$.

2.2. Geometrical setup and conditions. In order to provide pertinent computations, one assumes that the following condition is satisfied:

(C1) $\partial \Omega$ is a two-dimensional differentiable submanifold of $\mathbb{R}^{3}$, of class $\mathcal{C}^{2+\lambda}$.

This condition means that $\partial \Omega$ is locally the graph of a function $\varphi_{x}$ of class $\mathcal{C}^{2+\lambda}\left(\Pi_{x}, \mathbb{R}^{3}\right)$, where $\Pi_{x}$ is the tangential plan of $\partial \Omega$ in $x$. One denotes by $T_{x}$ : $\mathbb{R}^{3} \rightarrow \mathbb{R}^{3}$ the affine operator that changes 0 into $x$ and $\mathbb{R}^{2} \times\{0\}$ into $\Pi_{x}$.

Consequently, for any $x \in \partial \Omega$, there exists a function $\varphi_{x}$ defined in a neighborhood $A_{x}$ of 0 satisfying $\varphi_{x}(0)=0$ and $\nabla \varphi_{x}(0)=0$ such that

$$
T_{x}\left(A_{x} \times \varphi_{x}\left(A_{x}\right)\right) \subset \partial \Omega .
$$

One can notice that the bijective application $T_{x}$ is a composition of a translation and a rotation; thus its Jacobian is identically equal to 1 , and consequently one can integrate indifferently over $\partial \Omega$ or over $T_{x}^{-1} \partial \Omega$.

This sets up notation for the local description of $\partial \Omega$ around $x$ as a graph of functions $\varphi_{x}$ of class $C^{2+\lambda}\left(\mathbb{R}^{2}, \mathbb{R}\right)$, as displayed in Figure 2. One recalls that being 
of class $C^{m+\lambda}\left(\mathbb{R}^{2}, \mathbb{R}\right)$, with $0<\lambda \leqslant 1$, means that $\varphi_{x}$ is of class $C^{m}\left(\mathbb{R}^{2}, \mathbb{R}\right)$ and all its $m$ th order partial derivatives are $\lambda$-Hölder continuous. This means that for any $x \in \partial \Omega$, there exist two constants $C_{x}$ and $C_{x}^{\prime}$ such that locally one has

$$
\left|\varphi_{x}(s)-\frac{1}{2}^{t} s K_{x} s\right| \leqslant C_{x}|s|^{2+\lambda} \text { and }\left|\nabla \varphi_{x}(s)-K_{x} s\right| \leqslant C_{x}^{\prime}|s|^{1+\lambda}
$$

where $K_{x}$ is the Hessian matrix of $\varphi_{x}$ in $0=T_{x}^{-1}(x)$.

One then requires the following conditions on the globality of bounds defined above:

(C2) There exists $R>0$ such that domain definition $A_{x}$ of applications $\varphi_{x}$ contains $\mathcal{B}_{2}(0, R)$ for all $x \in \partial \Omega$.

(C3) The spectral radius of the Hessian $K_{x}$ of $\phi_{x}$ in 0 is bounded independently of $x \in \partial \Omega$, and its upper bound is denoted $\rho_{0}$.

(C4) There exist two constants $C$ and $C^{\prime}$ such that for any $x \in \partial \Omega$, one has $C_{x} \leqslant C$ and $C_{x}^{\prime} \leqslant C^{\prime}$.

Note that these hypotheses do not imply a lack of generality but provide some restrictions on smoothness (condition $(\mathrm{C} 1)$ ) and mapping orientation and size (conditions $(\mathrm{C} 2)$ and $(\mathrm{C} 4)$ ). One can also notice that $\partial \Omega$ being the boundary of an open set $\Omega$ and condition (C1) imply that $\partial \Omega$ is an oriented manifold and thus the existence of an inward normal field $n$ to $\partial \Omega$. Condition (C3) eliminates, for example, spiraloids or clothoidal surfaces whose curvature tends to infinity.

For convenience, one sets up the notation $\phi_{x}=\operatorname{Id}_{\mathbb{R}^{2}} \times \varphi_{x}$, which gives that

$$
S_{x} \equiv T_{x} \phi_{x}\left(\mathcal{B}_{2}(0, R)\right) \subset \partial \Omega .
$$

This local parameterization of $\partial \Omega$ gives the following integration formula:

$$
\int_{S_{x}} f(\xi) \mathrm{d} \sigma(\xi)=\int_{\mathcal{B}_{2}(0, R)} f \circ T_{x} \circ \phi_{x}(s)\left|N_{x}(s)\right| \mathrm{d} s,
$$

where

$$
N_{x}(s)=\frac{\partial \phi_{x}}{s_{1}}(s) \wedge \frac{\partial \phi_{x}}{s_{2}}(s)
$$

is the normal field induced by the parameterization $\phi_{x}$, its Euclidean norm being the Jacobian of the parameterization and satisfying

$$
\left|N_{x}(s)\right|=\sqrt{1+\left|\nabla \varphi_{x}(s)\right|},
$$

where $|\cdot|$ denotes the Euclidean norm in $\mathbb{R}^{3}$. Note that $T_{x}$ is not involved in this Jacobian because $\left|\partial_{s_{1}} T_{x} \circ \phi_{x} \wedge \partial_{s_{1}} T_{x} \circ \phi_{x}\right|=\left|\partial_{s_{1}} \phi_{x} \wedge \partial_{s_{1}} \phi_{x}\right|=\left|N_{x}(s)\right|$.

A few direct consequences of conditions $(\mathrm{C} 1)-(\mathrm{C} 4)$ can then be stated.

Proposition 2.3. Under conditions $(\mathrm{C} 1)-(\mathrm{C} 4)$, one has the following:

(P1) $N_{x}$, the Jacobian of the parameterization by $\phi_{x}$, is bounded independently of $x$, and its bound is denoted $M_{1}$.

(P2) There exists a constant $M_{2}$ such that

$$
\left|(x-\xi) \cdot n_{x}\right| \leqslant M_{2}|x-\xi|^{2} \quad \forall x, \xi \in \partial \Omega .
$$

Copyright $@$ by SIAM. Unauthorized reproduction of this article is prohibited. 
Proof. Let $x$ be a point of $\partial \Omega$. By (2.3), one gets

$$
\left|\nabla \varphi_{x}(s)\right| \leqslant\left|K_{x} s\right|+C_{x}^{\prime}|s|^{1+\lambda} \leqslant \rho_{0} R+C^{\prime} R^{1+\lambda} .
$$

Setting $M_{1}=\sqrt{1+\left(\rho_{0}+C^{\prime} R^{\lambda}\right)^{2} R^{2}}$ finishes the proof of assertion (P1).

Furthermore, for all $\xi$ in $\partial \Omega \cap \mathcal{B}_{3}(x, R) \subset T_{x} \phi_{x}\left(\mathcal{B}_{2}(0, R)\right)$ where $R$ is defined by condition (C2), one has $\xi=T_{x} \phi_{x}(s)$ with $\phi_{x}=\operatorname{Id}_{\mathbb{R}^{2}} \times \varphi_{x}$, and $T_{x}^{-1} n(x)=-e_{3}$. Consequently, one has $(\xi-x) \cdot n(x)=\varphi_{x}(s)-\varphi_{x}(0)=\varphi_{x}(s)$ and by condition (C3) and by definition of class $\mathcal{C}^{2+\lambda}$, one gets

$$
|(\xi-x) \cdot n(x)|=\left|\varphi_{x}(s)\right| \leqslant \frac{\rho_{0}}{2}|s|^{2}+C|s|^{2+\lambda} \leqslant\left(\frac{\rho_{0}}{2}+C R^{\lambda}\right)|s|^{2} .
$$

For any $\xi$ not in $\partial \Omega \cap \mathcal{B}_{3}(x, R)$, one has $\left|(\xi-x) \cdot n_{x}\right| \leqslant|\xi-x| \leqslant(\xi-x)^{2} / R$. Setting $M_{2}=\max \left(C R^{\lambda}+\rho_{0} / 2,1 / R\right)$ finishes the proof.

Part (P1) of the proposition will often be useful for calculus through the maps, while part (P2) implies that the operator $\widetilde{\mathcal{H}}(x, t) f$ is bounded over $\partial \Omega \times[0, T]$, which in itself is useful for showing that the $\widetilde{\mathcal{H}}$ is Hölder continuous.

Moreover, one needs a last condition of measure growth of $\partial \Omega$ :

(C5) There exist two positive constants $C$ and $k$ such that for any $x \in \partial \Omega$, the measure of the part of $\partial \Omega$ in the spherical strips $\mathcal{B}_{3}(x,(n+1) R) \backslash \mathcal{B}_{3}(x, n R)$ does not grow faster than $e^{k n^{2}}$; that is,

$$
\sigma(\{\xi \in \partial \Omega / n R \leqslant|\xi-x|<(n+1) R\}) \leqslant C e^{k n^{2}} \quad \forall x \in \partial \Omega .
$$

Condition (C5) is satisfied as soon as $\partial \Omega$ is compact, and provides useful majorations and controllable error estimates of map restriction when $\partial \Omega$ is not compact.

As a concluding note on conditions (C1)-(C5), let us remark that they are not very restrictive and do not lead to a lack of generality on the kind of surfaces considered. Their most significant effect is that they limit size and orientation of the maps and provide a good environment for integral calculus in the next sections.

2.3. Error estimation of the restriction to a map. The definition of $\widetilde{\mathcal{H}}$ involves a Gaussian function whose standard deviation tends to zero when $t$ tends to zero (smaller than $\sqrt{2 \nu t}$ ). Even if a Gaussian is not compactly supported, it decreases quickly, and its significant values are very localized. Consequently, thanks to limiting the final time $T$ of the heat equation (which is not a limitation in practice), we can consider the integral over one map,

$$
S_{x} \equiv T_{x} \phi_{x}\left(\mathcal{B}_{2}(0, R)\right) \subset \partial \Omega \subset \mathbb{R}^{3},
$$

instead of the whole surface $\partial \Omega$, and provide an error analysis thanks to the fast decreasing of Gaussian functions. One can introduce the heat layer restricted to $S_{x} \subset \partial \Omega$ as

$$
\widetilde{\mathcal{H}}_{S_{x}}(x, t) f=\nu \int_{0}^{t} \int_{S_{x}} \mathcal{L}_{x} G_{\xi, \tau}(x, t) f(\xi, \tau) \mathrm{d} \sigma(\xi) \mathrm{d} \tau
$$

for any continuous and bounded function $f: \partial \Omega \times[0, T] \rightarrow \mathbb{R}$, and the error due to the restriction on $S_{x} \subset \partial \Omega$ as

$$
\widetilde{\mathcal{H}}_{e r r}(x, t) f=\left(\widetilde{\mathcal{H}}(x, t)-\widetilde{\mathcal{H}}_{S_{x}}(x, t)\right) f=\nu \int_{0}^{t} \int_{\partial \Omega \backslash S_{x}} \mathcal{L}_{x} G_{\xi, \tau}(x, t) f(\xi, \tau) \mathrm{d} \sigma(\xi) \mathrm{d} \tau .
$$


By setting $\eta=\nu(t-\tau)$, one gets

$$
\frac{\left|\widetilde{\mathcal{H}}_{e r r}(x, t) f\right|}{\|f\|_{\infty}} \leqslant \int_{0}^{\nu t} \int_{\partial \Omega \backslash S_{x}}\left|\beta(x)-\frac{(x-\xi) \cdot n(x)}{2 \eta}\right| \widehat{G}(x-\xi, \eta) \mathrm{d} \sigma(\xi) \mathrm{d} \eta .
$$

One can now show that the error due to the restriction to a map can then be neglected at any order. The following integral calculus features the parametric approach of the present paper.

First, by condition (C2) there exists $R$, such that for all $x \in \partial \Omega$, one has $s=$ $T_{x}^{-1}(\xi)$ for all $\xi$ in the neighborhood $S_{x}$ of $x$ and the inequality

$$
|s|^{2} \leqslant|s|^{2}+\varphi_{x}(s)^{2}=\left|T_{x} \phi_{x}(s)-T_{x}(0)\right|^{2}=|\xi-x|^{2}<R^{2}
$$

and, consequently, the inclusions

$$
\partial \Omega \cap \mathcal{B}_{3}(x, R) \subset T_{x} \phi_{x}\left(\mathcal{B}_{2}(0, R)\right)=S_{x} \subset \partial \Omega
$$

which imply directly

$$
\partial \Omega \backslash S_{x}=\partial \Omega \backslash T_{x} \phi_{x}\left(\mathcal{B}_{2}(0, R)\right) \subset \partial \Omega \backslash \mathcal{B}_{3}(x, R) \subset \partial \Omega .
$$

Error estimate $\left|\widetilde{\mathcal{H}}_{e r r}(x, t) f\right|$ is thus majorated by

$$
\|f\|_{\infty} \int_{0}^{\nu t} \int_{\partial \Omega \backslash \mathcal{B}_{3}(x, R)}\left|\beta(x)-\frac{(x-\xi) \cdot n(x)}{2 \eta}\right| \widehat{G}(x-\xi, \eta) \mathrm{d} \sigma(\xi) \mathrm{d} \eta,
$$

which implies, due to part (P2) of Proposition 2.3, the following majoration:

$$
\|f\|_{\infty} \int_{0}^{\nu t}\left(|\beta(x)|+M_{2} \frac{|x-\xi|^{2}}{2 \eta}\right) \int_{\partial \Omega \backslash \mathcal{B}_{3}(x, R)} \widehat{G}(x-\xi, \eta) \mathrm{d} \sigma(\xi) \mathrm{d} \eta .
$$

Second, and this notation will be used throughout the paper, one has for all positive $x$ and $\alpha$,

$$
x^{\alpha} e^{-x} \leqslant L_{\alpha} e^{-x / 2} \quad \text { with } \quad L_{\alpha}=(2 \alpha)^{\alpha} e^{-\alpha} .
$$

It follows from (2.13) that expression (2.12) is majorated by

$$
\|f\|_{\infty}\left(\beta_{0}+L_{1} M_{2}\right) \int_{0}^{\nu t} \int_{\partial \Omega \backslash \mathcal{B}_{3}(x, R)} \frac{e^{-|x-\xi|^{2} / 8 \eta}}{(4 \pi \eta)^{3 / 2}} \mathrm{~d} \sigma(\xi) \mathrm{d} \eta .
$$

If $\partial \Omega$ is compact, then (2.14) is majorated by

$$
\|f\|_{\infty}\left(\beta_{0}+L_{1} M_{2}\right) \sigma(\partial \Omega) \int_{0}^{\nu t} \frac{e^{-R^{2} / 8 \eta}}{(4 \pi \eta)^{3 / 2}} \mathrm{~d} \eta
$$

which is finally majorated by

$$
\|f\|_{\infty} 2 \sqrt{2} L_{3 / 2} \frac{\beta_{0}+M_{2} L_{1}}{R^{3} \pi^{3 / 2}} e^{-R^{2} / 16 \nu t} \nu t \sigma(\partial \Omega) .
$$

Copyright $@$ by SIAM. Unauthorized reproduction of this article is prohibited. 
If $\partial \Omega$ is not compact, there is more work to be done, and one has to consider condition (C5) on maximal admissible growth of measures of $\partial \Omega$ contained in successive spherical strips, which leads to the same result. Indeed, one has the disjoint decomposition

$$
\partial \Omega=\left(\partial \Omega \cap \mathcal{B}_{3}(x, R)\right) \cup\left[\bigcup_{n \in \mathbb{N}^{*}}\left(\partial \Omega \cap\left(\mathcal{B}_{3}(0,(n+1) R) \backslash \mathcal{B}_{3}(0, n R)\right)\right)\right] ;
$$

thus

$$
\partial \Omega \backslash \mathcal{B}_{3}(x, R)=\bigcup_{n \in \mathbb{N}^{*}}\left(\partial \Omega \cap\left(\mathcal{B}_{3}(0,(n+1) R) \backslash \mathcal{B}_{3}(0, n R)\right)\right),
$$

and one can build a majoration of (2.14) by the use of condition (C5):

$$
\|f\|_{\infty} C \int_{0}^{\nu t}\left(\beta_{0}+L_{1} M_{2}\right)\left(\sum_{j \in \mathbb{N}^{*}} \frac{e^{-j^{2} R^{2} / 8 \eta}}{8 \pi^{3 / 2} \eta^{3 / 2}} e^{j^{2} k}\right) \mathrm{d} \eta
$$

with $C$ depending only on $\partial \Omega$. Equation (2.17) is itself majorated for $\nu t$ sufficiently small, i.e., for $\nu t \leqslant R^{2} / 8 k$, where $k$ depends only on $\partial \Omega$, by

$$
\|f\|_{\infty} C\left(\beta_{0}+L_{1} M_{2}\right) \int_{0}^{\nu t} \frac{1}{8 \pi^{3 / 2} \eta^{3 / 2}}\left(\sum_{j \in \mathbb{N}^{*}} \exp \left\{-j^{2}\left(\frac{R^{2}}{8 \eta}-k\right)\right\}\right) \mathrm{d} \eta .
$$

Now noticing that $j \leqslant j^{2}$ and that $k-R^{2} / 8 \eta \leqslant-R^{2} / 16 \eta$ for $\nu t \leqslant R^{2} / 16 k$, one gets another majoration of (2.18) by

$$
\|f\|_{\infty} C\left(\beta_{0}+L_{1} M_{2}\right) \int_{0}^{\nu t} \frac{1}{8 \pi^{3 / 2} \eta^{3 / 2}} \frac{1}{1-e^{-R^{2} / 16 \eta}} e^{-R^{2} / 16 \eta} \mathrm{d} \eta .
$$

Noticing also that $e^{-R^{2} / 16 \eta} \leqslant 1 / 2$ for $\eta \leqslant R^{2} / 16 \ln 2$, one gets

$$
\|f\|_{\infty} C\left(\beta_{0}+L_{1} M_{2}\right) \int_{0}^{\nu t} \frac{e^{-R^{2} / 16 \eta}}{4 \pi^{3 / 2} \eta^{3 / 2}} \mathrm{~d} \eta
$$

which gives the final majoration for $\nu t \leqslant R^{2} / 16 k^{a}$ st with $k^{*}=\max (\ln 2, k)$, i.e., for $t$ sufficiently small:

$$
\|f\|_{\infty} 16 C L_{3 / 2} \frac{\beta_{0}+L_{1} M_{2}}{\pi^{3 / 2} R^{3}} e^{-R^{2} / 32 \nu t} \nu t .
$$

Equation (2.16) holds when $\partial \Omega$ is a compact manifold, and is extended to the noncompact case by means of (2.21). From these equations, one gets the following proposition.

Proposition 2.4. Under conditions $(\mathrm{C} 1)-(\mathrm{C} 5)$ and previous notation, there exists a constant $C$ independent of $x$ and $t$ such that

$$
\frac{\left|\widetilde{\mathcal{H}}_{e r r, \mathcal{B}_{2}(\zeta, R)}(x, t) f\right|}{\|f\|_{\infty}} \leqslant \frac{\left|\widetilde{\mathcal{H}}_{e r r, A}(x, t) f\right|}{\|f\|_{\infty}} \leqslant C\|f\|_{\infty} e^{-R^{2} / 32 \nu t} \nu t=\mathcal{O}\left(t^{\infty}\right)
$$

for $t$ sufficiently small and for all $x \in \partial \Omega$ and any $f \in \mathbb{L}^{\infty}(\partial \Omega)$.

Copyright $@$ ㅇ by SIAM. Unauthorized reproduction of this article is prohibited. 


$$
\begin{aligned}
& -\frac{1}{2} \widetilde{\mu}(x, t)+\widetilde{\mathcal{H}}(x, t) \widetilde{\mu}=F(x, t) \quad \stackrel{\text { param. }}{\longrightarrow} \mu_{x}^{b}(s, t)=\widetilde{\mu}\left(T_{x} \phi_{x}(s), t\right) \\
& \downarrow \text { restr. on } S_{x} \\
& -\frac{1}{2} \mu^{\natural}(x, t)+\widetilde{\mathcal{H}}_{S_{x}}(x, t) \mu^{\natural}=F(x, t) \stackrel{\text { param. }}{\longrightarrow} \mu_{x}(s, t)=\mu^{\natural}\left(T_{x} \phi_{x}(s), t\right) \\
& \Uparrow \\
& -\frac{1}{2} \mu_{x}(0, t)+\mathcal{H}(x, t) \mu_{x}=F(x, t) \\
& \downarrow \text { approx. on } \overline{S_{x}} \\
& -\frac{1}{2} \bar{\mu}_{x}(0, t)+\overline{\mathcal{H}}(x, t) \bar{\mu}_{x}=F(x, t) \stackrel{\text { param. }}{\longrightarrow} \bar{\mu}_{x}(s, t)=\mu^{\sharp}\left(T_{x} \phi_{x}(s), t\right) .
\end{aligned}
$$

FIG. 3. Different integrodifferential operators and associated integral equations and densities involved in the surface potential analysis.

3. Geometrical approximation. It has been shown in section 2.3 that only a local analysis of the heat layer is required to obtain the development in early time of the heat layer at any order, since the Gaussian kernel has significant values very locally.

The local parameterizations of the manifold and its quadratic osculating manifold are set up in sections 3.1 and 3.2, respectively, and we give a few approximation lemmas in section 3.3 that will be useful in proving convergence in section 3.4 and providing error estimates of truncations to get the final results.

The process of parameterization and approximation can be split into two different steps as shown in Figure 3, which should be read as follows. First one considers the original integral equation of solution $\widetilde{\mu}$, whose parameterization is denoted $\mu_{x}^{b}$ in a neighborhood of origin whose image is a neighborhood $S_{x} \subset \partial \Omega$ of $x$.

The map-restricted heat layer $\widetilde{\mathcal{H}}_{S_{x}}$ is introduced in section 3.1 and defines a new integral equation whose solution is denoted $\mu^{\natural}$ and its parameterization $\mu_{x}$, which is itself the solution of an integral equation involving $\mathcal{H}$, the restricted heat layer acting on parameterizations, i.e., such that

$$
\widetilde{\mathcal{H}}_{S_{x}}(x, t) \mu^{\natural}=\mathcal{H}(x, t) \mu_{x} .
$$

The portion of surface $S_{x}$ is then approximated by its best quadratic approximant $\overline{S_{x}}$ in section 3.2, which induces a heat layer on the surface approximant denoted $\overline{\mathcal{H}}$, and another integral equation involving this operator acting on parameterizations, whose solution is denoted $\bar{\mu}_{x}: A \rightarrow \mathbb{R}$. This solution corresponds to a density $\mu^{\sharp}: \partial \Omega \rightarrow \mathbb{R}$.

While the relation between densities $\widetilde{\mu}$ and $\mu^{\natural}$ is quite obvious by means of proposition 2.4, the link between $\mu^{\natural}$ and $\mu^{\sharp}$ is less obvious and requires an analysis of approximation relations between the associated integral operators. The convergence of densities is established in section 3.4, especially in Proposition 3.6.

3.1. Local parameterization of the surface. The two-dimensional manifold $\partial \Omega$ is described by its maps, which are themselves defined by means of the description of $\partial \Omega$ by graphs of functions $\varphi_{x}$, given in (2.4), which reads as follows:

$$
T_{x} \phi_{x} \equiv T_{x}\left(\operatorname{Id}_{\mathbb{R}^{2}} \times \varphi_{x}\right): A=\mathcal{B}(0, R) \subset \mathbb{R}^{2} \longrightarrow T_{x} \phi_{x}(A) \equiv S_{x} \subset \partial \Omega .
$$


A density $\widetilde{f}: \partial \Omega \rightarrow \mathbb{R}$ can be restricted on a map as

$$
\begin{aligned}
\tilde{f}: S_{x} \subset \partial \Omega \subset \mathbb{R}^{3} \times[0, T] & \longrightarrow \mathbb{R} \times[0, T], \\
(\xi, t) & \longmapsto \widetilde{f}(\xi, t) .
\end{aligned}
$$

One can introduce the local parameterization $f$ in space of $\tilde{f}$ through $T_{x} \phi_{x}$ in a neighborhood $S_{x}$ of $x$ in $\partial \Omega$, so that $\xi=T_{x} \phi_{x}(s)$, which gives

$$
\begin{aligned}
& f: A \times[0, T] \quad \longrightarrow \quad \mathbb{R} \times[0, T], \\
& (s, t) \quad \longmapsto f(s, t)=\widetilde{f}\left(T_{x} \circ \phi_{x}(s), t\right) \\
& =\tilde{f}\left(T_{x}\left(s_{1}, s_{2}, \varphi_{x}(s)\right), t\right),
\end{aligned}
$$

where $A \equiv \mathcal{B}_{2}(0, R) \subset \mathbb{R}^{2}$.

One also considers the double heat layer restricted to a map acting on parameterizations of densities, defined consequently by

$$
\mathcal{H}(x, t) f=\widetilde{\mathcal{H}}_{S_{x}}(x, t) \tilde{f}=\nu \int_{0}^{t} \int_{A} \mathcal{L}_{x} G_{T_{x} \phi_{x}(s), \tau}(x, t) f(s, \tau)\left|N_{x}(s)\right| \mathrm{d} s \mathrm{~d} \tau,
$$

where

$$
N_{x}(s)=\frac{\partial \phi_{x}}{s_{1}}(s) \wedge \frac{\partial \phi_{x}}{s_{2}}(s)
$$

and $|N(s)|$ denotes its Euclidean norm in $\mathbb{R}^{3}$. This Jacobian calculus holds since the Jacobian of $T_{x}$ is identically equal to 1 and gives

$$
\left|N_{x}(s)\right|=\sqrt{1+\left|\nabla \varphi_{x}(s)\right|^{2}}
$$

which is bounded with respect to $x$ over $\partial \Omega$ by Proposition 2.3(P1). One can notice that the Gaussian functions are spherically symmetric; thus the equalities

$$
\begin{aligned}
G_{T_{x} \phi_{x}(s), \tau}(x, t) & =G_{\phi_{x}(s), \tau}\left(T_{x}^{-1} x, t\right)=G_{\phi_{x}(s), \tau}(0, t) \\
& =G_{0,0}\left(\phi_{x}(s), t-\tau\right)=\widehat{G}\left(\phi_{x}(s), \nu(t-\tau)\right) \\
& =\frac{e^{-\left(s^{2}+\varphi_{x}(s)^{2}\right) / 4 \nu(t-\tau)}}{(4 \pi \nu(t-\tau))^{3 / 2}}
\end{aligned}
$$

hold because $\phi_{x}=\operatorname{Id}_{\mathbb{R}^{2}} \times \varphi_{x}$.

Since one has $\varphi_{x}(0)=0$ and $\nabla \varphi_{x}(0)=0$, this leads, by means of $(2.3)$, to

$$
\varphi_{x}(s)=\frac{1}{2}{ }^{t} s K_{x} s+\mathcal{O}\left(s^{2+\lambda}\right),
$$

where $K_{x}$ is the symmetric $2 \times 2$ curvature matrix of $\partial \Omega$ in $x$, in the spirit of the two-dimensional approach of [24].

3.2. Local approximation of the surface. In order to provide a local analysis on an explicitly known surface, and to estimate the error related to geometrical approximation, one considers the second order approximation of $\partial \Omega$ in a neighborhood $S_{x}$ of $x \in \partial \Omega$. This means we introduce the quadratic form

$$
\bar{\varphi}_{x}(s)=\frac{1}{2}{ }^{t} s K_{x} s
$$

and all the related quantities, as displayed in Figure 4: 


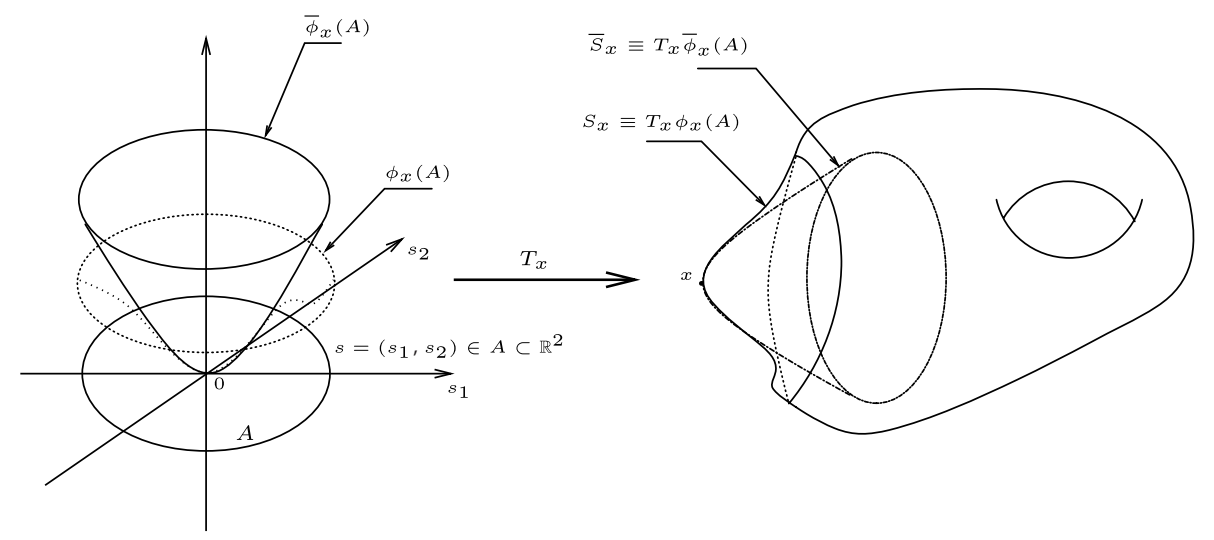

FIG. 4. Context and notation of surface approximation.

- $\bar{\phi}_{x}=T_{x} \circ\left(\operatorname{Id}_{\mathbb{R}^{2}} \times \bar{\varphi}_{x}\right)$,

- $\overline{S_{x}}=T_{x} \bar{\phi}_{x}(A)$, the quadratic approximant of $S_{x}$,

- $\overline{\mathcal{H}}(x, t)$, the integral operator defined over $\overline{S_{x}}$,

- $\bar{N}_{x}(s)=\partial_{s_{1}} \bar{\phi}_{x} \wedge \partial_{s_{2}} \bar{\phi}_{x}$, the Jacobian of this parameterization, which verifies

$$
\left|\bar{N}_{x}(s)\right|=\sqrt{1+\left|\nabla \varphi_{x}(s)\right|^{2}}=\sqrt{1+\left|K_{x} s\right|^{2}},
$$

- $\bar{\mu}_{x}: A \rightarrow \mathbb{R}$, the parameterized solution of the integral equation

$$
-\bar{\mu}_{x}(0, t)+2 \overline{\mathcal{H}}(x, t) \bar{\mu}_{x}=F(x, t),
$$

which defines the density $\mu^{\sharp}: \partial \Omega \rightarrow \mathbb{R}$ by $\mu^{\sharp}(x, t)=\bar{\mu}_{x}(0, t)$.

Furthermore, the boundary operator $\mathcal{L}_{x}$ defining the Robin-Fourier condition can be written as $\beta(x) \operatorname{Id}+n(x) \cdot \nabla$. The gradient of the Gaussian kernel satisfies

$$
\nabla_{x} G_{\xi, \tau}(x, t)=-\frac{x-\xi}{2 \nu(t-\tau)} G_{\xi, \tau}(x, t)
$$

thus

$$
\mathcal{L}_{x} G_{\xi, \tau}(x, t)=\left(\beta(x)-\frac{(x-\xi) \cdot n(x)}{2 \nu(t-\tau)}\right) G_{\xi, \tau}(x, t)
$$

Moreover, the gradient $\nabla \varphi_{x}(0)=0$ implies $T_{x}^{-1} n(x)=-e_{3}$, and since the scalar product is conserved by rotation/translation (i.e., by operator $T_{x}$ ), one gets

$$
\begin{aligned}
-(x-\xi) \cdot n(x) & =\left(T_{x} \phi_{x}(s)-T_{x} \phi_{x}(0)\right) \cdot n(x) \\
& =\left(\operatorname{Id}_{\mathbb{R}^{2}} \times \varphi_{x}\right)(s) \cdot\left(-b e_{3}\right)=-\varphi_{x}(s)
\end{aligned}
$$

for all $\xi=T_{x} \phi_{x}(s)=T_{x}\left(\operatorname{Id}_{\mathbb{R}^{2}} \times \varphi_{x}\right)(s) \in S_{x}$, i.e., for all $s \in A=\mathcal{B}_{2}(0, R)$.

For a function $f: A \rightarrow \mathbb{R}$, one has consequently

$$
\begin{gathered}
\mathcal{H}(x, t) f= \\
\nu \int_{0}^{t} \int_{A}\left(\beta(x)-\frac{\varphi_{x}(s)}{2 \nu(t-\tau)}\right) G_{0, \tau}\left(\phi_{x}(s), t\right) f\left(T_{x} \phi_{x}(s), \tau\right) \sqrt{1+\left|\nabla \varphi_{x}(s)\right|^{2}} \mathrm{~d} s \mathrm{~d} \tau
\end{gathered}
$$

Copyright (c) by SIAM. Unauthorized reproduction of this article is prohibited. 
and similarly, one gets

$$
\begin{gathered}
\overline{\mathcal{H}}(x, t) f= \\
\nu \int_{0}^{t} \int_{A}\left(\beta(x)-\frac{\bar{\varphi}_{x}(s)}{2 \nu(t-\tau)}\right) G_{0, \tau}\left(\bar{\phi}_{x}(s), t\right) f\left(T_{x} \phi_{x}(s), \tau\right) \sqrt{1+\left|\nabla \bar{\varphi}_{x}(s)\right|^{2}} \mathrm{~d} s \mathrm{~d} \tau .
\end{gathered}
$$

For practical considerations in the estimation of the integrals defined above and their related quantities, one will often need a purely computational result, which is given to increase readability.

Lemma 3.1 (integral majoration). There exists a constant $C_{0}$ independent of $t$ such that for $R \geqslant 0$ and $k>0$, the majoration

$$
\Gamma_{\alpha, \eta}^{R, k}(\tau)=\int_{0}^{\tau} \int_{R}^{\infty} \frac{r^{\alpha}+u^{\alpha / 2}}{u^{\eta}} e^{-r^{2} / k u} r \mathrm{~d} r \mathrm{~d} u \leqslant C_{0} e^{-R^{2} / 2 k \tau} \tau^{2-\eta+\alpha / 2}
$$

holds when $\eta-\alpha / 2<2$, i.e., when the integral is convergent.

Consequently, $\Gamma_{\alpha, \eta}^{R, k}(\tau)=\mathcal{O}\left(\tau^{2-\eta+\alpha / 2}\right)$ if $R=0$, and $\Gamma_{\alpha, \eta}^{R, k}(\tau)=\mathcal{O}\left(\tau^{\infty}\right)$ otherwise.

Proof. One has

$$
r \frac{r^{\alpha}+u^{\alpha / 2}}{u^{\eta}} e^{-r^{2} / k u} \leqslant C r u^{\alpha / 2-\eta} e^{-r^{2} / 2 k u}
$$

with $C=1+L_{\alpha / 2} k^{\alpha / 2}$, and consequently

$$
\begin{aligned}
\int_{R}^{\infty} \frac{r^{\alpha}+u^{\alpha / 2}}{u^{\eta}} e^{-r^{2} / k u} r \mathrm{~d} r & \leqslant C k u^{\alpha / 2+1-\eta} \int_{R}^{\infty} \frac{2 r}{2 k u} e^{-r^{2} / 2 k u} \mathrm{~d} r \\
& =C k u^{\alpha / 2+1-\eta} e^{-R^{2} / 2 k u}
\end{aligned}
$$

Integral (3.1) is then majorated by

$$
\Gamma_{\alpha, \eta}^{R, k}(\tau) \leqslant C k \int_{0}^{\tau} u^{\alpha / 2+1-\eta} e^{-R^{2} / 2 k u} \mathrm{~d} u \leqslant C_{0} e^{-R^{2} / 2 k \tau} \tau^{2-\eta+\alpha / 2}
$$

with

$$
C_{0}=\frac{k C}{2-\eta+\alpha / 2}>0 .
$$

The comment on order is then obvious.

The question now is how great an error is made in the integral operator when the surface $\partial \Omega$ is approximated by its best osculating quadratic surface, and how the error on the integral operator is related to the error on the solution $\mu_{x}$.

3.3. Approximation lemma. In this section we exhibit in Lemma 3.5 the convergence rate of $\overline{\mathcal{H}}$ toward $\mathcal{H}$ in time variable when the surface $\partial \Omega$ is replaced by its best quadratic approximation. This lemma requires preliminary results in Lemmas 3.2 and 3.1. The result of Lemma 3.5 is used in the next sections and also implies the convergence of $\bar{\mu}_{x}$ toward $\mu_{x}$ with the same rate, as explained in Proposition 3.6.

One can define the usual norms considered herein. The simple norm $|\cdot|$ denotes the Euclidean norm of $\mathbb{R}^{3}$, and the maximum double norm denotes the usual $\mathbb{L}^{\infty}$ norm over $\partial \Omega$ or $\partial \Omega \times[0, T]$, depending on the context.

Copyright $@$ by SIAM. Unauthorized reproduction of this article is prohibited. 
The triple norm of linear operator applies to the integrodifferential operators defined above. Indeed, for any $(x, t) \in \partial \Omega \times[0, T], \mathcal{H}(x, t)$ is a linear operator that verifies $|\mathcal{H}(x, t) f| \leqslant\|f\|_{\infty}|\mathcal{H}(x, t) 1|$ for any bounded function $f$, with equality for $f$ identically equal to 1 . One then gets

$$
\||\mathcal{H}(x, t)|\|_{\infty}=\sup _{f \in \mathbb{L} \infty(A) \backslash\{0\}} \frac{|\mathcal{H}(x, t) f|}{\|f\|_{\infty}}=\sup _{\|f\|_{\infty}=1}|\mathcal{H}(x, t) f|=|\mathcal{H}(x, t) 1| .
$$

The triple norm $\left.\|\overline{\mathcal{H}}(x, t)\|\right|_{\infty}$ is itself a function of $x$ and $t$, whose $\mathbb{L}^{\infty}$ norm over $\partial \Omega$ is defined as

$$
\|\| \mathcal{H}(\cdot, t)\left|\|\|_{\infty}=\|x \mapsto\|\|\mathcal{H}(x, t)\|\left\|_{\infty}\right\|_{\infty}=\sup _{x \in \partial \Omega}\left\|\overline{\mathcal{H}}(x, t)\left|\|_{\infty}=\sup _{x \in \partial \Omega}\right| \mathcal{H}(x, t) 1 \mid .\right.\right.
$$

Lemma 3.2. Let $f_{t}: A \times[0, T] \rightarrow \mathbb{R}$ be a function, possibly depending on $t \in[0, T]$, such that there exist two constants $\alpha \geqslant 0$ and $C_{f}$ independent of $t$ satisfying

$$
\left|f_{t}(s, \tau)\right| \leqslant C_{f}\left(|s|^{\alpha}+|\nu(t-\tau)|^{\alpha / 2}\right)
$$

for all $(s, \tau) \in A \times[0, t]$. Under the hypothesis $(\mathrm{C} 1)-(\mathrm{C} 4)$ of section 2.2 , with $\mathcal{H}$ defined by formula (3.10), there exists a constant $C_{0}$ such that for any $x \in \partial \Omega$, one has

$$
\mathcal{H}(x, t) f_{t}=C_{0} t^{(\alpha+1) / 2} .
$$

Proof. The portion of surface $S_{x}$ is the image by the isometry $T_{x}$ of the graph of $\varphi_{x}$ over the two-dimensional ball $A=\mathcal{B}_{2}(0, R)$, with

$$
\varphi_{x}(s)=\frac{1}{2}{ }^{t} s K_{x} s+\mathcal{O}\left(s^{2+\lambda}\right)
$$

since $\partial \Omega$ is a manifold of class $\mathcal{C}^{2+\lambda}$. One has, through the map $T_{x} \phi_{x}$,

$$
\mathcal{H}(x, t) f_{t}=\nu \int_{0}^{t} \int_{A}\left(\beta(x)-\frac{\varphi_{x}(s)}{2 \nu(t-\tau)}\right) G_{0, \tau}\left(\phi_{x}(s), t\right) f(s, \tau)\left|N_{x}(s)\right| \mathrm{d} s \mathrm{~d} \tau
$$

with

$$
\left|N_{x}(s)\right|=\sqrt{1+\left|\nabla \varphi_{x}(s)\right|^{2}} \leqslant M_{1}
$$

and

$$
\left|\varphi_{x}(s)\right| \leqslant\left|\frac{1}{2}{ }^{t} s K_{x} s\right|+C|s|^{2+\lambda} \leqslant\left(\frac{\rho_{0}}{2}+C R^{\lambda}\right) s^{2},
$$

where $M_{1}, \rho_{0}$, and $C$ are the constants introduced, respectively, in Proposition 2.3(P1), condition (C3), and condition (C4).

One gets consequently, assuming $C_{1}=\left(\rho_{0} / 2+C R^{\lambda}\right)$ and $u=\nu t$,

$$
\begin{aligned}
& \left|\mathcal{H}(x, t) f_{t}\right| \leqslant \\
& M_{1} \int_{0}^{\nu t} \int_{A}\left(\beta_{0}+C_{1} \frac{|s|^{2}}{2 u}\right) \frac{1}{(4 \pi u)^{3 / 2}} \exp \left(-\frac{|s|^{2}+\left({ }^{t} s K_{x} s\right)^{2}}{4 u}\right) f_{t}(s, \tau) \mathrm{d} s \mathrm{~d} \tau .
\end{aligned}
$$

Copyright $@$ by SIAM. Unauthorized reproduction of this article is prohibited. 
Now noticing that $\left({ }^{t} s K s\right)^{2} \geqslant 0$, assuming $r=|s|$, and applying the Hölder-like hypothesis on $f_{t}$, one has an axisymmetric expression that integrates into

$$
\left|\mathcal{H}(x, t) f_{t}\right| \leqslant \frac{2 C_{f} M_{1}}{\sqrt{\pi}} \int_{0}^{\nu t} \int_{0}^{R}\left(\beta_{0}+C_{1} \frac{r^{2}}{2 u}\right) \frac{r^{\alpha}+u^{\alpha / 2}}{(4 u)^{3 / 2}} e^{-r^{2} / 4 u} r \mathrm{~d} r \mathrm{~d} \tau .
$$
3.1:

Using relation (2.13) that gives $x e^{-x} \leqslant L_{1} e^{-x / 2}$, the result follows from Lemma

$$
\left|\mathcal{H}(x, t) f_{t}\right| \leqslant \frac{C_{f} M_{1}}{4 \sqrt{\pi}}\left(\beta_{0}+2 L_{1} C_{1}\right) \Gamma_{\alpha, 3 / 2}^{0,8}(t)=\mathcal{O}\left(t^{1 / 2+\alpha / 2}\right) .
$$

Note that $C_{0}=C_{f} M_{1}\left(\beta_{0}+2 L_{1} C_{1}\right) / 4 \sqrt{\pi}$ depends neither on $x \in \partial \Omega$ nor on $t \in$ $[0, T]$.

One first has to notice that this result holds when $\alpha=0$, which provides a useful majoration of $\mathcal{H}(x, t) f$ when $f$ is only bounded, that is to say, majoration of $\mathcal{H}(x, t) 1$, and directly gives that

$$
\|\mid \mathcal{H}(x, t)\| \|_{\infty}=O\left(t^{1 / 2}\right)
$$

for any $(x, t) \in \partial \Omega \times[0, T]$.

One can also notice that the final majorant in the proof is not dependent on $x$, and applying this again to the unity function, one can extend result (3.18) into

$$
\||| \mathcal{H}(\cdot, t)\|\left\|_{\infty}=\right\| x \mapsto\|\mid \mathcal{H}(x, t)\|_{\infty} \|_{\infty}=O\left(t^{1 / 2}\right) .
$$

Another important result is that the lemma also holds for $\overline{\mathcal{H}}(x, t)$, which is a special case with the function $\varphi_{x}=\bar{\varphi}_{x}$ of class $\mathcal{C}^{\infty}$ so a fortiori $\mathcal{C}^{3}$, with a constant $C$ set to 0 in (3.15). This gives that there exists a constant $C$ such that

$$
\|\| \overline{\mathcal{H}}(\cdot, t) \mid\|\|_{\infty} \leqslant C t^{1 / 2}
$$

and consequently the following corollary.

Corollary 3.3. Let $\overline{\mathcal{H}}$ be the operator defined by formula (3.11). Then, under the hypothesis of Lemma 3.2 , for $t$ sufficiently small, one has \|\|$\overline{\mathcal{H}}(\cdot, t)\|\|_{\infty}<1 / 2$.

From Lemma 3.2, one also has the following property valid on $S_{x} \subset \partial \Omega$.

Corollary 3.4. Let $Z_{x, t}$ be a function over $S_{x} \times[0, T]$ such that there exist two constants $\alpha \geqslant 0$ and $C_{Z}$ independent of $x, y, t$, and $\tau$ satisfying

$$
\left|Z_{x, t}(y, \tau)\right| \leqslant C_{Z}\left(|x-y|^{\alpha}+|\nu(t-\tau)|^{\alpha / 2}\right)
$$

for all $(y, \tau) \in S_{x} \times[0, t]$ and $(x, t) \in \partial \Omega \times[0, T]$. Under the hypothesis $(\mathrm{C} 1)-(\mathrm{C} 4)$ of section 2.2 , there exists a constant $C_{0}$ such that for any $x \in \partial \Omega$, one has

$$
\widetilde{\mathcal{H}}_{S_{x}}(x, t) Z_{x, t}=C_{0} t^{(\alpha+1) / 2} .
$$

Proof. Let $Z_{x, t}^{\natural}$ be the parameterization of $Z$ through the map $T_{x} \phi_{x}$ :

$$
Z_{x, t}^{\natural}(s, \tau)=Z_{x, t}\left(T_{x} \phi_{x}(s), \tau\right),
$$

which gives $\widetilde{\mathcal{H}}_{S_{x}}(x, t) Z_{x, t}=\mathcal{H}(x, t) Z_{x, t}^{\natural}$. One then has

$$
\left|Z_{x, t}^{\natural}(s, \tau)\right| \leqslant C_{Z}\left(\left|T_{x} \phi_{x}(s)-x\right|^{\alpha}+|\nu(t-\tau)|^{\alpha / 2}\right) \leqslant C_{0}\left(|s|^{\alpha}+|\nu(t-\tau)|^{\alpha / 2}\right)
$$

since $T_{x}$ is an isometry and $\left|\phi_{x}(s)\right|^{2}=|s|^{2}+\left|\varphi_{x}(s)\right|^{2} \leqslant\left(1+C_{1}^{2} R^{2}\right)|s|^{2}$, which leads to set $C_{0}=C_{Z} \sqrt{1+C_{1}^{2} R^{2}}$. One can then apply Lemma 3.2.

Copyright $@$ by SIAM. Unauthorized reproduction of this article is prohibited. 
3.4. Geometrical convergence. Now we present the following stability lemma of fundamental importance.

Lemma 3.5 ( $\mathcal{H}-\overline{\mathcal{H}}$ estimation). Under the hypothesis $(\mathrm{C} 1)-(\mathrm{C} 4)$ of section 2.2 , $\partial \Omega$ being a two-dimensional manifold of class $\mathcal{C}^{2+\lambda}, 0<\lambda \leqslant 1$, there exists a constant $C_{0}$ independent of $t$ such that

$$
\|\| \mathcal{H}(\cdot, t)-\left.\overline{\mathcal{H}}(\cdot, t)\|\|\right|_{\infty}=\sup _{x \in \partial \Omega}\|\| \mathcal{H}(x, t)-\overline{\mathcal{H}}(x, t)\|\|_{\infty} \leqslant C_{0} t^{(1+\lambda) / 2} .
$$

Proof. The proof of this lemma uses more or less the same technique as the proof of Lemma 3.2. One chooses an $x \in \partial \Omega$ and gets for a bounded function over $S_{x} \subset \partial \Omega$

$$
|\mathcal{H}(x, t) f-\overline{\mathcal{H}}(x, t) f| \leqslant\|f\|_{\infty}|\mathcal{H}(x, t) 1-\overline{\mathcal{H}}(x, t) 1|
$$

with equality for $f \equiv 1$, and consequently

$$
\||\mathcal{H}(x, t)-\overline{\mathcal{H}}(x, t)|\|_{\infty}=|\mathcal{H}(x, t) 1-\overline{\mathcal{H}}(x, t) 1| .
$$

It remains to build an accurate majoration of this quantity independent of $x$.

Operators $\mathcal{H}(x, t)$ and $\overline{\mathcal{H}}(x, t)$ are defined by formulas (3.10) and (3.11), which give

$$
\||\mathcal{H}(x, t)-\overline{\mathcal{H}}(x, t)|\|_{\infty}=\left|\int_{0}^{\nu t} \int_{A} \gamma(s, u) \widehat{G}\left(\phi_{x}(s), u\right)-\bar{\gamma}(s, u) \widehat{G}\left(\bar{\phi}_{x}(s), u\right) \mathrm{d} s \mathrm{~d} u\right|,
$$

where $u=\nu(t-\tau)$, and

$$
\gamma(s, u)=\left(\beta_{x}-\frac{\varphi_{x}(s)}{2 u}\right) \sqrt{1+\left|\nabla \varphi_{x}(s)\right|^{2}}
$$

and

$$
\bar{\gamma}(s, u)=\left(\beta_{x}-\frac{\bar{\varphi}_{x}(s)}{2 u}\right) \sqrt{1+\left|\nabla \bar{\varphi}_{x}(s)\right|^{2}}
$$

Moreover, $\widehat{G}\left(\bar{\phi}_{x}(s), u\right)$ and $\widehat{G}\left(\phi_{x}(s), u\right)$ are both majorated by $e^{-|s|^{2} / 4 u} /(4 \pi u)^{3 / 2}$, and thus

$$
\||\mathcal{H}(x, t)-\overline{\mathcal{H}}(x, t)|\|_{\infty} \leqslant \int_{0}^{\nu t} \int_{\mathbb{R}^{2}}|\gamma(s, u)-\bar{\gamma}(s, u)| \frac{e^{-|s|^{2} / 4 u}}{(4 \pi u)^{3 / 2}} \mathrm{~d} s \mathrm{~d} u .
$$

The graph approximation, by means of condition (C4), satisfies

$$
\left|\varphi_{x}(s)-\bar{\varphi}_{x}(s)\right| \leqslant C|s|^{2+\lambda} \text {. }
$$

Moreover, the gradients satisfy $\nabla \bar{\varphi}_{x}(s)=K_{x} s$, and there exists a constant $C^{\prime}$ such that $\left|\nabla \varphi_{x}(s)-K_{x} s\right| \leqslant C^{\prime}|s|^{1+\lambda}$; thus

$$
\left|\nabla \varphi_{x}(s)-\nabla \bar{\varphi}_{x}(s)\right|^{2} \leqslant C^{\prime}|s|^{2+2 \lambda} .
$$

One can do the following decomposition relying on the triangular inequality:

$$
|\gamma(s, u)-\bar{\gamma}(s, u)| \leqslant K_{1}+K_{2}
$$

Copyright $@$ by SIAM. Unauthorized reproduction of this article is prohibited. 
with

$$
K_{1}=\left|\left(\beta_{x}-\frac{\varphi_{x}(s)}{2 u}\right) \sqrt{1+\left|\nabla \varphi_{x}(s)\right|^{2}}-\left(\beta_{x}-\frac{\varphi_{x}(s)}{2 u}\right) \sqrt{1+\left|\nabla \bar{\varphi}_{x}(s)\right|^{2}}\right|
$$

and

$$
K_{2}=\left|\left(\beta_{x}-\frac{\varphi_{x}(s)}{2 u}\right) \sqrt{1+\left|\nabla \bar{\varphi}_{x}(s)\right|^{2}}-\left(\beta_{x}-\frac{\bar{\varphi}_{x}(s)}{2 u}\right) \sqrt{1+\left|\nabla \bar{\varphi}_{x}(s)\right|^{2}}\right| .
$$

The first part $K_{1}$ can be majorated by

$$
K_{1} \leqslant\left(\beta_{0}+\left|\frac{\varphi_{x}(s)}{2 u}\right|\right)\left|\sqrt{1+\left|\nabla \varphi_{x}(s)\right|^{2}}-\sqrt{1+\left|\nabla \bar{\varphi}_{x}(s)\right|^{2}}\right|,
$$

and the second by

$$
K_{2} \leqslant \frac{1}{2 u}\left|\varphi_{x}(s)-\bar{\varphi}_{x}(s)\right| \sqrt{1+\left|\nabla \bar{\varphi}_{x}(s)\right|^{2}},
$$

which, noticing that $\left|\nabla \varphi_{x}(s)\right|=\left|K_{x} s\right| \leqslant \rho_{0} R \leqslant \rho_{0} R+C^{\prime} R^{1+\lambda}$, gives (see proof of Proposition 2.3(P1))

$$
K_{2} \leqslant M_{1} \frac{\left|\varphi_{x}(s)-\bar{\varphi}_{x}(s)\right|}{2 u} \leqslant M_{1} C^{\prime} \frac{|s|^{2+\lambda}}{2 u} .
$$

Furthermore, the triangular inequality

$$
\left|\nabla \varphi_{x}(s)\right|^{2} \leqslant\left|\nabla \bar{\varphi}_{x}(s)\right|^{2}+\left|\nabla \varphi_{x}(s)-\nabla \bar{\varphi}_{x}(s)\right|^{2}
$$

implies

$$
\sqrt{1+\left|\nabla \varphi_{x}(s)\right|^{2}} \leqslant \sqrt{1+\left|\nabla \bar{\varphi}_{x}(s)\right|^{2}+\left|\nabla \varphi_{x}(s)-\nabla \bar{\varphi}_{x}(s)\right|^{2}}
$$

Now one can notice that the epigraph of $-\sqrt{x}$ is convex; thus for any $a>0$ one has $\sqrt{a+x} \leqslant \sqrt{a}+x / 2 \sqrt{a}$ for all $x \in[-a,+\infty[$. This implies

$$
\sqrt{1+\left|\nabla \varphi_{x}(s)\right|^{2}} \leqslant \sqrt{1+\left|\nabla \bar{\varphi}_{x}(s)\right|^{2}}+\frac{\left|\nabla \varphi_{x}(s)-\nabla \bar{\varphi}_{x}(s)\right|^{2}}{2 \sqrt{1+\left|\nabla \bar{\varphi}_{x}(s)\right|^{2}}} .
$$

This leads to a majoration of (3.24) using (3.23):

$$
K_{1} \leqslant \frac{C^{\prime}}{2}\left(\beta_{0}+\left|\frac{\varphi_{x}(s)}{2 u}\right|\right)|s|^{2+2 \lambda} .
$$

Since one has $\left|\varphi_{\zeta}(s)\right| \leqslant\left(\rho_{0}+C R^{\lambda}\right)|s|^{2}$, one finally has the majoration

$$
|\gamma(s, u)-\bar{\gamma}(s, u)| \leqslant \frac{C^{\prime}}{2}\left(\beta_{0}+\left(\rho_{0}+C R^{\lambda}\right) \frac{|s|^{2}}{2 u}\right)|s|^{2+2 \lambda}+M_{1} C^{\prime} \frac{|s|^{2+\lambda}}{2 u} .
$$

Integral (3.21) can then be majorated by applying Lemma 3.1 and majoration obtained by (3.30). Indeed, one can set $r=|s|$ and apply Lemma 3.1 with $R=0$. This gives

Copyright (c) by SIAM. Unauthorized reproduction of this article is prohibited. 


$$
\begin{aligned}
\|\mid \mathcal{H}(x, t)-\overline{\mathcal{H}}(x, t)\|_{\infty} & \leqslant \frac{C^{\prime} \beta_{0}}{8 \sqrt{\pi}} \Gamma_{2+2 \lambda, 3 / 2}^{0,4}(t)+\frac{C^{\prime} \beta_{0}}{16 \sqrt{\pi}}\left(\rho_{0}+C R^{\lambda}\right) \Gamma_{4+2 \lambda, 5 / 2}^{0,4}(t) \\
& +\frac{M_{1} C^{\prime}}{8 \sqrt{\pi}} \Gamma_{2+\lambda, 5 / 2}^{0,4}(t)=\mathcal{O}\left(t^{(1+\lambda) / 2}\right)
\end{aligned}
$$

Proposition 3.6 (geometrical convergence). Under the previous notation and conditions $(\mathrm{C} 1)-(\mathrm{C} 4)$, with $\mathcal{H}$ and $\overline{\mathcal{H}}$ defined, respectively, by formulas (3.10) and (3.11), and for $t$ sufficiently small, the densities $\mu^{\natural}$ and $\mu^{\sharp}$ defined in section 3 satisfy

$$
\frac{\left\|\mu^{\natural}(\cdot, t)-\mu^{\sharp}(\cdot, t)\right\|_{\infty}}{\left\|\mu^{\natural}(\cdot, t)\right\|_{\infty}} \leqslant \frac{2}{1-2 \mid\|\overline{\mathcal{H}}(\cdot, t)\|\|\|_{\infty}}\|\| \mathcal{H}(\cdot, t)-\overline{\mathcal{H}}(\cdot, t) \mid\|\|_{\infty} .
$$

Proof. Let $\mu^{\natural}$, introduced in section 3, be the solution of the integral equation involving the localized integrodifferential operator (see also Figure 3),

$$
-\frac{1}{2} \mu^{\natural}(x, t)+\widetilde{\mathcal{H}}_{S_{x}} \mu^{\natural}=F(x, t),
$$

whose solution $\mu^{\natural}$, bounded over $\partial \Omega$, has a local parameterization $\mu_{x}$ on $A$ by $\mu_{x}(s, t)=$ $\mu^{\natural}\left(T_{x} \phi_{x}(s), t\right)$. The integral equation (3.33) is then equivalent to

$$
-\frac{1}{2} \mu_{x}(0, t)+\mathcal{H}(x, t) \mu_{x}=F(x, t) .
$$

The $S_{x}$-localized integrodifferential operator $\mathcal{H}$ acting on parameterized densities can then be defined on an approximated surface $\overline{S_{x}}$, which leads to the integral equation

$$
-\frac{1}{2} \bar{\mu}_{x}(0, t)+\overline{\mathcal{H}}(x, t) \bar{\mu}_{x}=F(x, t)
$$

of solution $\bar{\mu}_{x}: A \times[0, T] \rightarrow \mathbb{R}$, which is the parameterization of the density $\mu^{\sharp}(x, t)=$ $\bar{\mu}_{x}(0, t)$. The functions $\mu_{x}$ and $\bar{\mu}_{x}$ are both defined on the same domain $A$ but represent densities on two different surfaces, $S_{x} \subset \partial \Omega$ and $\overline{S_{x}}$.

One can then write, subtracting (3.34) and (3.35),

$$
\begin{aligned}
\frac{\mu_{x}(s, t)-\bar{\mu}_{x}(s, t)}{2} & =\mathcal{H}(x, t) \mu_{x}-\overline{\mathcal{H}}(x, t) \bar{\mu}_{x} \\
& =\mathcal{H}(x, t) \mu_{x}-\overline{\mathcal{H}}(x, t) \mu_{x}+\overline{\mathcal{H}}(x, t) \mu_{x}-\overline{\mathcal{H}}(x, t) \bar{\mu}_{x}
\end{aligned}
$$

and

(3.36)

$$
\frac{\left|\mu_{x}(s, t)-\bar{\mu}_{x}(s, t)\right|}{2} \leqslant|||\mathcal{H}(x, t)-\overline{\mathcal{H}}(x, t)|\left\|_{\infty}\right\| \mu_{x}\left\|_{\infty}+\right\| \overline{\mathcal{H}}(x, t)\|\|_{\infty}\left\|\mu_{x}-\bar{\mu}_{x}\right\|_{\infty},
$$

where the $\mathbb{L}^{\infty}$-norm of densities is taken over $A$. One can notice that

$$
\left\|\mu_{x}\right\|_{\infty}=\sup _{s \in A}\left|\mu_{x}(s)\right|=\sup _{\xi \in S_{x}}\left|\mu^{\natural}(\xi)\right| \leqslant \sup _{\xi \in \partial \Omega}\left|\mu^{\natural}(\xi)\right|=\left\|\mu^{\natural}\right\|_{\infty}
$$

and also that $\left\|\mu_{x}-\bar{\mu}_{x}\right\|_{\infty} \leqslant\left\|\mu^{\natural}-\mu^{\sharp}\right\|_{\infty}$.

Copyright (c) by SIAM. Unauthorized reproduction of this article is prohibited. 
Taking the maximum of $x$ over $\partial \Omega$ for the triple norms in (3.36) and applying it to $s=0$ gives

$$
\left|\mu^{\natural}(x, t)-\mu^{\sharp}(x, t)\right| \leqslant 2\left|\left\||| \mathcal{H}(\cdot, t)-\overline{\mathcal{H}}(\cdot, t)\left|\|\|_{\infty}\left\|\mu^{\natural}\right\|_{\infty}+2\right||||\overline{\mathcal{H}}(\cdot, t)| \mid\right\|_{\infty}\left\|\mu^{\natural}-\mu^{\sharp}\right\|_{\infty} .\right.
$$

The right-hand side of (3.37) is independent of $x$; thus one can take the maximum of $x$ over $\partial \Omega$ on the left-hand side and get

$$
\left(1-2\left\||| \overline{\mathcal{H}}(\cdot, t)|\||_{\infty}\right)\left\|\mu^{\natural}-\mu^{\sharp}\right\|_{\infty} \leqslant 2||\|\mid \mathcal{H}(\cdot, t)-\overline{\mathcal{H}}(\cdot, t)\|\|\|_{\infty}\left\|\mu^{\natural}\right\|_{\infty} .\right.
$$

Finally, by Corollary 3.3 of Lemma 3.2 , one has $\||| \overline{\mathcal{H}}(\cdot, t) \mid\| \|_{\infty}<1 / 2$, which finishes the proof.

This proposition leads directly to the order of convergence when $\partial \Omega$ is sufficiently smooth, when used with Lemma 3.5

COROLlary 3.7. Under the hypothesis of Proposition 3.6, if $\partial \Omega$ is a manifold of class $\mathcal{C}^{2+\lambda}, 0<\lambda \leqslant 1$, then there exists a constant $C_{0}$ independent of $t$ such that

$$
\left\|\mu^{\natural}(\cdot, t)-\mu^{\sharp}(\cdot, t)\right\|_{\infty} \leqslant C t^{(1+\lambda) / 2} .
$$

This means that a manifold of class $\mathcal{C}^{3}$ allows us to reach order 1 in density convergence when locally approximating the surface by its best quadratic osculating surface.

4. Leading order of $\overline{\mathcal{H}}$. We have shown in last section that the best parabolic approximation of the surface leads at least to first order in the approximation of the density, which is enough to carry out the main contribution of the curvature effect to the solution.

LEMma 4.1. Under conditions $(\mathrm{C} 1)-(\mathrm{C} 4)$ and previous notation, we consider the integral

$$
\mathcal{H}_{0}(x, t)=\int_{0}^{\nu t} \int_{\mathbb{R}^{2}}\left(\beta_{x}-\frac{{ }^{t} s K_{x} s}{4 u}\right) \frac{e^{-\left(|s|^{2}+{ }^{t} s K_{x} s / 2\right) / 4 u}}{(4 \pi u)^{3 / 2}} \mathrm{~d} s \mathrm{~d} u .
$$

Then $\overline{\mathcal{H}}(x, t) 1=\mathcal{H}_{0}(x, t)+\mathcal{O}\left(t^{3 / 2}\right)$, with $\overline{\mathcal{H}}(x, t)$ defined by $(3.11)$.

Proof. We first introduce the integral

$$
H_{1}(x, t)=\int_{0}^{\nu t} \int_{\mathbb{R}^{2}}\left(\beta_{x}-\frac{{ }^{t} s K_{x} s}{4 u}\right) \frac{e^{-\left(|s|^{2}+\left({ }^{t} s K_{x} s\right)^{2} / 4\right) / 4 u}}{(4 \pi u)^{3 / 2}} \sqrt{1+\left|K_{x} s\right|^{2}} \mathrm{~d} s \mathrm{~d} u
$$

with ${ }^{t} s K_{x} s / 2=\bar{\varphi}_{x}(s)$. We then get

$$
\left|\overline{\mathcal{H}}(x, t) 1-H_{1}(x, t)\right| \leqslant \frac{M_{1}}{4 \sqrt{\pi}} \int_{0}^{\nu t} \int_{R}^{+\infty}\left(\beta_{0}+\frac{\left|\bar{\varphi}_{x}(s)\right|}{2 u}\right) \frac{e^{-|s|^{2} / 4 u}}{u^{3 / 2}} r \mathrm{~d} r,
$$

where $\left|\bar{\varphi}_{x}(s)\right| \leqslant \rho_{0}|s|^{2} / 2$, which gives

$$
\left|\overline{\mathcal{H}}(x, t) 1-H_{1}(x, t)\right| \leqslant \frac{M_{1}}{4 \sqrt{\pi}}\left(\beta_{0}+\rho_{0} L_{1}\right) \Gamma_{0,3 / 2}^{R, 8}(t) .
$$

One can now focus on the estimation of $\mathcal{H}_{1}(x, t)-H_{0}(x, t)$. The difference between the two operators lies in the Jacobian $N_{x}(s)$, which is not present in the definition of $\mathcal{H}_{0}(x, t)$. One has

$$
\left|\mathcal{H}_{1}(x, t)-H_{0}(x, t)\right| \leqslant \frac{\beta_{0}+\rho_{0} L_{1}}{4 \sqrt{\pi}} \int_{0}^{\nu t} \int_{0}^{+\infty} \frac{e^{-|s|^{2} / 8 u}}{u^{3 / 2}}\left(\sqrt{1+\rho_{0}^{2} r^{2}}-1\right) r \mathrm{~d} r,
$$


which gives, since $\sqrt{1+\alpha} \leqslant 1+\alpha / 2$ for any $\alpha \geqslant-1$,

$$
\left|\mathcal{H}_{1}(x, t)-H_{0}(x, t)\right| \leqslant \frac{\rho_{0}^{2}}{8 \sqrt{\pi}}\left(\beta_{0}+\rho_{0} L_{1}\right) \Gamma_{2,3 / 2}^{0,8}(t) .
$$

Applying triangular inequality and noticing that $\Gamma_{0,3 / 2}^{R, 8}(t)+\Gamma_{2,3 / 2}^{0,8}(t)=\mathcal{O}\left(t^{3 / 2}\right)$ finishes the proof the lemma.

Now that the integrals $\overline{\mathcal{H}}(x, t) 1$ and $\mathcal{H}_{0}(x, t)$ are linked and are an approximation of one another at an order higher than surface approximation (see Lemma 3.5), one can focus on the estimation of $\mathcal{H}_{0}(x, t)$.

Proposition 4.2. Under conditions $(\mathrm{C} 1)-(\mathrm{C} 4)$ and $\mathcal{H}_{0}(x, t)$ defined by (4.1), one has uniformly

$$
\mathcal{H}_{0}(x, t)=\left(\beta_{x}-\frac{\operatorname{tr}\left(K_{x}\right)}{2}\right) \sqrt{\frac{\nu t}{\pi}}+\mathcal{O}\left(t^{3 / 2}\right) .
$$

Proof. We begin to write the curvature matrix $K_{x}$ as

$$
K_{x}=\left[\begin{array}{ll}
\kappa_{1} & \kappa_{0} \\
\kappa_{0} & \kappa_{2}
\end{array}\right]
$$

By means of the cylindrical change of variable $s=(r \cos \theta, r \sin \theta)$, the integral (4.1) becomes

$$
\mathcal{H}_{0}(x, t)=\int_{0}^{2 \pi} \int_{0}^{\nu t} \int_{0}^{\infty}\left(\beta_{x}-\frac{r^{2} m(\theta)}{4 u}\right) \frac{e^{-\left(r^{2}+r^{4} m(\theta)^{2} / 4\right) / 4 u}}{(4 \pi u)^{3 / 2}} r \mathrm{~d} r \mathrm{~d} u \mathrm{~d} \theta
$$

where $m(\theta)=\kappa_{1} \cos ^{2} \theta+\kappa_{2} \sin ^{2} \theta+\kappa_{0} \cos \theta \sin \theta$. Now posing $(r, u)=\left(\xi \zeta, \zeta^{2} / 4\right)$, associated with a Jacobian $\zeta^{2} / 2$, one gets

$$
\mathcal{H}_{0}(x, t)=\frac{1}{2 \pi^{3 / 2}} \int_{0}^{2 \pi} \int_{0}^{2 \sqrt{\nu t}} \int_{0}^{\infty} \gamma(\xi, \zeta, \theta, x, t) \mathrm{d} \xi \mathrm{d} \zeta \mathrm{d} \theta
$$

with

$$
\gamma(\xi, \zeta, \theta, x, t)=\left(\xi \beta_{x}-\xi^{3} m(\theta)\right) \exp \left\{-\xi^{2}\left(1+\frac{\xi^{2} \zeta^{2} m(\theta)^{2}}{4}\right)\right\},
$$

which is infinitely differentiable in variables $\xi, \zeta$, and $\theta$.

Since for any positive constants $a$ and $b$, the function $f(\zeta)=e^{-a-b \zeta^{2}}$ satisfies $f^{\prime}(0)=0$ and $f^{\prime \prime}$ is bounded over $\mathbb{R}^{+}$, there exists $\zeta_{0} \in[0, \zeta]$ such that

$$
f(\zeta)=f(0)+\zeta^{2} f^{\prime \prime}\left(\zeta_{0}\right) / 2 .
$$

In order to make a Taylor development of $\gamma$ in $\zeta$ valid, one has to exhibit the bounds of $f^{\prime \prime}$ with respect to coefficients $a, b$, and $c$. Indeed, one has

$$
f^{\prime \prime}(\zeta)=\left(4 b^{2} \zeta^{2}-2 b\right) e^{-a-b \zeta^{2}}
$$

thus

$$
\left|f^{\prime \prime}(\zeta)\right| \leqslant 2 b e^{-a}\left(1+2 b \zeta^{2}\right) e^{-b \zeta^{2}} \leqslant 4 b e^{-a} .
$$

Copyright (c) by SIAM. Unauthorized reproduction of this article is prohibited. 
Applying this result with $a=\xi^{2}$ and $b=\xi^{4} m(\theta)^{2} / 4$ to the exponential part of (4.7), and using majoration (2.13), one gets

$$
\left|\exp \left\{-\xi^{2}\left(1+\frac{\xi^{2} \zeta^{2} m(\theta)^{2}}{4}\right)\right\}-1\right| \leqslant \zeta^{2} \xi^{4} m(\theta)^{2} e^{-\xi^{2}} \leqslant L_{2} \zeta^{2} m(\theta)^{2} e^{-\xi^{2} / 2} .
$$

Consequently, one has

$$
\left|\gamma(\xi, \zeta, \theta, x, t)-\left(\xi \beta_{x}-\xi^{3} m(\theta)\right) e^{-\xi^{2}}\right| \leqslant L_{2}\left(\beta_{0} \xi+|m(\theta)| \xi^{3}\right) \zeta^{2} m(\theta)^{2} e^{-\xi^{2} / 2},
$$

which is itself majorated, using again formula (2.13), by

$$
\sqrt{2} L_{2}\left(L_{1 / 2} \beta_{0}+2 L_{3 / 2}|m(\theta)|\right) \zeta^{2} m(\theta)^{2} e^{-\xi^{2} / 4} .
$$

Now noticing that

$$
\begin{aligned}
|m(\theta)| & \leqslant\left|\kappa_{1}\right|+\left|\kappa_{0}\right|+\left|\kappa_{0}\right|+\left|\kappa_{2}\right| \\
& \leqslant 2 \max \left(\left|\kappa_{1}\right|+\left|\kappa_{0}\right|,\left|\kappa_{0}\right|+\left|\kappa_{2}\right|\right)=2||\left|K_{x}\right|\left\|_{1} \leqslant 2 \sqrt{2}||\left|K_{x}\right|\right\|_{2} \leqslant 2 \sqrt{2} \rho_{0},
\end{aligned}
$$

where $|\|\cdot \mid\|$ is the standard norm for linear operators, and setting

$$
C=8 \sqrt{2} L_{2}\left(L_{1 / 2} \beta_{0}+4 L_{3 / 2} \rho_{0}\right) \rho_{0}^{2},
$$

one gets

$$
\left|\gamma(\xi, \zeta, \theta, x, t)-\left(\xi \beta_{x}-\xi^{3} m(\theta)\right) e^{-\xi^{2}}\right| \leqslant C \zeta^{2} e^{-\xi^{2} / 4},
$$

whose right-hand side is variable separated and integrates obviously into

$$
\int_{0}^{2 \pi} \int_{0}^{2 \sqrt{\nu t}} \int_{0}^{\infty} \zeta^{2} e^{-\xi^{2} / 4} \mathrm{~d} \xi \mathrm{d} \zeta \mathrm{d} \theta=\frac{16 \pi^{3 / 2}}{3}(\nu t)^{3 / 2}
$$

and consequently

$$
\left|\mathcal{H}_{0}(x, t)-\frac{\sqrt{\nu t}}{\pi^{3 / 2}} \int_{0}^{2 \pi} \int_{0}^{\infty}\left(\xi \beta_{x}-\xi^{3} m(\theta)\right) e^{-\xi^{2}} \mathrm{~d} \xi \mathrm{d} \theta\right| \leqslant \frac{8 C}{3}(\nu t)^{3 / 2} .
$$

Moreover, one has

$$
\int_{0}^{2 \pi} m(\theta) \mathrm{d} \theta=\pi \kappa_{1}+\pi \kappa_{2}=\pi \operatorname{tr}\left(K_{x}\right)
$$

and thus

$$
\left|\mathcal{H}_{0}(x, t)-\sqrt{\frac{\nu t}{\pi}} \int_{0}^{\infty}\left(2 \xi \beta_{x}-\xi^{3}\left(\kappa_{1}+\kappa_{2}\right)\right) e^{-\xi^{2}} \mathrm{~d} \xi\right| \leqslant \frac{8 C}{3}(\nu t)^{3 / 2}
$$

with

$$
\int_{0}^{\infty}\left(2 \xi \beta_{x}-\xi^{3}\left(\kappa_{1}+\kappa_{2}\right)\right) e^{-\xi^{2}} \mathrm{~d} \xi=\beta_{x}-\frac{\kappa_{1}+\kappa_{2}}{2},
$$

which concludes the proof.

Copyright $@$ by SIAM. Unauthorized reproduction of this article is prohibited. 
5. Curvature effect on the whole surface. From all the previous sections we can give now the following result.

THEOREM 5.1. Let $\Omega$ be an open set of $\mathbb{R}^{3}$ such that $\partial \Omega$ is a two-dimensional manifold of class $\mathcal{C}^{2+\lambda}, 0<\lambda \leqslant 1$, satisfying conditions $(\mathrm{C} 1)-(\mathrm{C} 5)$, and $\mathcal{L}_{x}$ is a Robin-Fourier differential operator $\mathcal{L}_{x}=\beta(x) \mathrm{Id}+n(x) \cdot \nabla$ with $\beta$ bounded over $\partial \Omega$ and $n(x)$ the inward normal to $\partial \Omega$ in $x$. Let also $\widetilde{\mathcal{H}}$ be the following integrodifferential operator:

$$
\widetilde{\mathcal{H}}(x, t) f=\nu \int_{0}^{t} \int_{\partial \Omega} \mathcal{L}_{x} G_{\xi, \tau}(x, t) f(\xi, \tau) \mathrm{d} \sigma(\xi) \mathrm{d} \tau
$$

for all continuous and bounded functions $f: \partial \Omega \times[0, T] \rightarrow \mathbb{R}$. If the density $\widetilde{\mu}$ is $\alpha$-Hölder continuous, then

$$
\widetilde{\mathcal{H}}(x, t) \widetilde{\mu}=[\widetilde{\mu}(x, t)(\beta(x)-\bar{\kappa}(x))] \sqrt{\frac{\nu t}{\pi}}+\mathcal{O}\left(t^{(1+\gamma) / 2)}\right),
$$

where $\gamma=\min (\alpha, \lambda)$ and $\bar{\kappa}(x)$ is the mean curvature of $\partial \Omega$ in $x$.

Proof. Let $\widetilde{\mu}$ be a density that is supposed $\alpha$-Hölder continuous with $\alpha \in] 0,1]$. One can introduce the function $Z_{x, t}$ defined by

$$
Z_{x, t}:(y, \tau) \mapsto Z_{x, t}(y, \tau)=\widetilde{\mu}(y, \tau)-\widetilde{\mu}(x, t)
$$

with the following property:

$$
\left|Z_{x, t}(y, \tau)\right|=|\widetilde{\mu}(y, \tau)-\widetilde{\mu}(x, t)| \leqslant C_{Z}\left(|x-y|^{\alpha}+|\nu(t-\tau)|^{\alpha / 2}\right) .
$$

One then gets

$$
\widetilde{\mathcal{H}}_{S_{x}}(x, t) Z_{x, t}=\widetilde{\mathcal{H}}_{S_{x}}(x, t) \widetilde{\mu}-\widetilde{\mu}(x, t) \widetilde{\mathcal{H}}_{S_{x}}(x, t) 1 .
$$

By Corollary 3.4 of Lemma 3.2, one gets that there exists a constant $C_{1}$ independent of $x$ and $t$ such that

$$
\left|\widetilde{\mathcal{H}}_{S_{x}}(x, t) Z_{x, t}\right|=\left|\widetilde{\mathcal{H}}_{S_{x}}(x, t) \widetilde{\mu}-\widetilde{\mu}(x, t) \widetilde{\mathcal{H}}_{S_{x}}(x, t) 1\right| \leqslant C_{1} t^{(\alpha+1) / 2}
$$

with $\widetilde{\mathcal{H}}_{S_{x}}(x, t) 1=\mathcal{H}(x, t) 1$, and thus

$$
\left|\widetilde{\mathcal{H}}_{S_{x}}(x, t) 1-\overline{\mathcal{H}}(x, t) 1\right| \leqslant C_{2} t^{(1+\lambda) / 2}
$$

by Lemma 3.5 ,

$$
\left|\overline{\mathcal{H}}(x, t) 1-\mathcal{H}_{0}(x, t)\right| \leqslant C_{3} t^{3 / 2}
$$

by Lemma 4.1 , and also

$$
\left|\mathcal{H}_{0}(x, t)-\left(\beta_{x}-\bar{\kappa}(x)\right) \sqrt{\nu t / \pi}\right| \leqslant C_{4} t^{3 / 2}
$$

by Proposition 4.2, where $\bar{\kappa}(x)=\operatorname{tr} K_{x} / 2$ is the mean curvature of $\partial \Omega$ in $x$.

Joining together (5.5), (5.6), (5.7), and (5.8) gives

$$
\left|\widetilde{\mathcal{H}}_{S_{x}}(x, t) \widetilde{\mu}-\left(\beta_{x}-\bar{\kappa}(x)\right) \widetilde{\mu}(x, t) \sqrt{\nu t / \pi}\right| \leqslant C_{5} t^{(\gamma+1) / 2}
$$

Copyright $@$ by SIAM. Unauthorized reproduction of this article is prohibited. 
with $\gamma=\min (\alpha, \lambda)$ and $C_{5}=C_{1} T^{(\alpha-\gamma) / 2}+C_{2} T^{(\lambda-\gamma) / 2}+\left(C_{3}+C_{4}\right) T^{2-\gamma / 2}$. Noticing that

$$
\widetilde{\mathcal{H}}(x, t) \widetilde{\mu}=\widetilde{\mathcal{H}}_{S_{x}}(x, t) \widetilde{\mu}+\mathcal{O}\left(t^{\infty}\right)
$$

by Proposition 2.4 gives the final result.

First of all, it is necessary to remark that Theorem 5.1 gives the early behavior of the solution, as mentioned in section 1.

Corollary 5.2. Let $\partial \Omega$ be a two-dimensional manifold of class $\mathcal{C}^{3}$ and let $F$ be a bounded function over $\partial \Omega \times[0, T]$ such that the solution $\widetilde{\mu}$ of the integral equation

$$
-\frac{1}{2} \widetilde{\mu}(x, t)+\widetilde{\mathcal{H}}(x, t) \widetilde{\mu}=F(x, t)
$$

is bounded and $(1-\varepsilon)$-Hölder continuous with the Hölder exponent satisfying $0 \leqslant \varepsilon<$ 1. Under the notation and hypothesis of Theorem 5.1, one has

$$
\widetilde{\mu}(x, t)=\frac{-2 F(x, t)}{1+2(\bar{\kappa}(x)-\beta(x)) \sqrt{\nu t / \pi}}+\mathcal{O}\left(t^{1-\varepsilon / 2}\right) .
$$

Furthermore, one can build a result in a smoother context concerning torsion-free surfaces.

THEOREM 5.3. Let $\Omega$ be an open set of $\mathbb{R}^{3}$ such that $\partial \Omega$ is a two-dimensional manifold of class $\mathcal{C}^{3+\lambda^{*}}$ without torsion, with $0<\lambda^{*} \leqslant 1$, satisfying conditions (C1)(C5). Let $\beta$ and $F$ be two functions bounded, respectively, over $\partial \Omega$ and $\partial \Omega \times[0, T]$ such that the solution $\tilde{\mu}$ of the integral equation (5.11) is of class $\mathcal{C}^{1+\alpha}(\partial \Omega \times[0, T])$ and bounded. Then the solution $\widetilde{\mu}$ of (5.11) satisfies

$$
\widetilde{\mathcal{H}}(x, t) \widetilde{\mu}=[\widetilde{\mu}(x, t)(\beta(x)-\bar{\kappa}(x))] \sqrt{\frac{\nu t}{\pi}}+\mathcal{O}\left(t^{1+\gamma / 2}\right),
$$

where $\gamma=\min \left(\alpha, \lambda^{*}\right)$ and $\bar{\kappa}(x)$ is the mean curvature of $\partial \Omega$ in $x$.

Proof. In the context of a manifold smoother than $\mathcal{C}^{3}$, there exist two constants $C_{x}$ and $C_{x}^{\prime}$ such that

$$
\left|\varphi_{x}(s)-\frac{1}{2}{ }^{t} s K_{x} s-\frac{1}{6} T_{x}^{i j k} s_{i} s_{j} s_{k}\right| \leqslant C_{x}|s|^{3+\lambda}
$$

and

$$
\left|\nabla \varphi_{x}(s)-K_{x} s-\frac{1}{2}{ }^{t} s\left(\mathcal{T}_{x}: e .\right) s\right| \leqslant C_{x}^{\prime}|s|^{2+\lambda},
$$

where $\mathcal{T}_{x}$ is the torsion tensor of $\varphi_{x}$ in 0 defined as

$$
\mathcal{T}_{x}^{i j k}=\frac{\partial^{3} \varphi_{x}}{\partial s_{i} \partial s_{j} \partial s_{k}}(0)
$$

and $\mathcal{T}_{x}^{i j k} s_{i} s_{j} s_{k}$ is its associated cubic form. The torsion tensor can be contracted with the vectors of canonical basis $e_{k}$, such that the $k$ th component of $\mathcal{T}_{x}: e$. is the matrix $\mathcal{T}_{x}: e_{k}$ associated to the quadratic form ${ }^{t} s\left(\mathcal{T}_{x}: e_{k}\right) s$. This can be equivalently stated, using again the Einstein notation, as

$$
{ }^{t} s\left(\mathcal{T}_{x}: e .\right) s=\left(\mathcal{T}_{x}: e .\right)^{i j} s_{i} s_{j}=\mathcal{T}_{x}^{i j k} s_{i} s_{j} e_{k} .
$$

Copyright (c) by SIAM. Unauthorized reproduction of this article is prohibited. 
This statement is also equivalent to the symmetric formulation $\left(E^{*}\right)^{\otimes 3} \equiv E \otimes E^{*} \otimes E^{*}$ with $E=\mathbb{R}^{2}$.

Constants $C_{x}$ and $C_{x}^{\prime}$ can be assumed to be bounded independently of $x$ over $\partial \Omega$, and in the case of a torsion-free surface (for example, cylinders and spheres are torsionfree), the torsion tensor is identically equal to 0 , which leads to majorations of the same kind as those of equations (2.3) with majorants $C|s|^{3+\lambda^{*}}$ and $C^{\prime}|s|^{2+\lambda^{*}}$, respectively, for the gradients. In this context, Lemma 3.5 holds with $\left.\left.\lambda=1+\lambda^{*} \in\right] 0,2\right]$, which gives that

$$
\widetilde{\mathcal{H}}(x, t) \widetilde{\mu}=\mathcal{H}(x, t) \mu_{x}^{b}+\mathcal{O}\left(t^{\infty}\right)=\overline{\mathcal{H}}(x, t) \mu_{x}^{b}+\mathcal{O}\left(t^{1+\lambda^{*} / 2}\right)
$$

with $\mu_{x}^{b}(s, \tau)=\widetilde{\mu}\left(T_{x} \phi_{x}(s), \tau\right)$.

Moreover, thanks to greater regularity of the density through the maps, one can write the following development:

$$
\begin{aligned}
\mu_{x}^{b}(s, \tau)= & \mu_{x}^{b}(0, t)+R_{x, t}(s, \tau)-\left.\nu(t-\tau) \frac{\partial \mu_{x}^{b}}{\partial \tau}\right|_{0, t} \\
& +\mathcal{O}\left(|s|^{1+\alpha}+|s| u^{\alpha / 2}\right)+\mathcal{O}\left(|s|^{\alpha} u+u^{1+\alpha / 2}\right)
\end{aligned}
$$

with $R_{x, t}(s, \tau)=s \cdot \nabla \mu_{x}^{b}(0, t)$ and $u=\nu|t-\tau|$.

By oddness, one has $\overline{\mathcal{H}}(x, t) R_{x, t}=0$, and one can easily establish upon proof of Lemma 3.2 that

$$
\left|\overline{\mathcal{H}}(x, t)\left[|s|^{a}(\nu(t-\tau))^{b}\right]\right| \leqslant \frac{2 M_{1}}{\sqrt{\pi}} \int_{0}^{\nu t} \int_{0}^{R}\left(\beta_{0}+\frac{\rho_{0}}{2} \frac{r^{2}}{2 u}\right) \frac{r^{a} u^{b} e^{-r^{2} / 4 u}}{8 u^{3 / 2}} r \mathrm{~d} r \mathrm{~d} \tau
$$

which gives

$$
\left|\overline{\mathcal{H}}(x, t)\left[|s|^{a}(\nu(t-\tau))^{b}\right]\right| \leqslant C_{1} \Gamma_{a, 3 / 2-b}^{0,8}=\mathcal{O}\left(t^{1 / 2+b+a / 2}\right)
$$

with $C_{1}=M_{1}\left(\beta_{0}+L_{1} \rho_{0}\right) / 4 \sqrt{\pi}$. This implies that $|\overline{\mathcal{H}}(x, t)[\nu(t-\tau)]|=\mathcal{O}\left(t^{3 / 2}\right)$; thus (5.17) reads as

$$
\mu_{x}^{b}(s, \tau)=\mu_{x}^{b}(0, t)+\mathcal{O}\left(t^{\eta}\right)
$$

with $\eta=\min (3 / 2,1+\alpha / 2,3 / 2+\alpha / 2)=1+\alpha / 2$. Combining this with (5.16) gives $(5.21)$

$$
\widetilde{\mathcal{H}}(x, t) \widetilde{\mu}=\overline{\mathcal{H}}(x, t) \mu_{x}^{b}+\mathcal{O}\left(t^{1+\lambda^{*} / 2}\right)=\mu_{x}^{b}(0, t) \overline{\mathcal{H}}(x, t) 1+\mathcal{O}\left(t^{1+\lambda^{*} / 2}\right)+\mathcal{O}\left(t^{1+\alpha / 2}\right) .
$$

By Lemma 4.1 and Proposition 4.2, one has

$$
\left|\overline{\mathcal{H}}(x, t) 1-\left(\beta_{x}-\bar{\kappa}(x)\right) \sqrt{\nu t / \pi}\right|=\mathcal{O}\left(t^{3 / 2}\right),
$$

and consequently (5.21) leads to

$$
\widetilde{\mathcal{H}}(x, t) \widetilde{\mu}=\widetilde{\mu}(x, t) \overline{\mathcal{H}}(x, t) 1+\mathcal{O}\left(t^{1+\gamma / 2}\right)=\widetilde{\mu}(x, t)\left(\beta_{x}-\bar{\kappa}(x)\right) \sqrt{\nu t / \pi}+\mathcal{O}\left(t^{1+\gamma / 2}\right)
$$

with $\gamma=\min \left(\lambda^{*}, \alpha\right)$.

Note that Theorem 5.3 is not an extension of Theorem 5.1, because a function can be more regular than Hölder continuous (even with an exponent 1) and less than $\mathcal{C}^{1}$ (for example, $\sqrt{t}$ ). Moreover, one can notice that this result is useful only as a 
kind of regularity estimation of the integrodifferential operator $\widetilde{\mathcal{H}}$, since the density obtained as the solution of the original integral equation (5.11) exhibits a square-root singularity in the general case (see Corollary 5.2) and consequently cannot be $\mathcal{C}^{1}$.

Furthermore, a direct corollary of this result is that if the density is $\mathcal{C}^{2}(\partial \Omega \times[0, T])$, which is in practice a restrictive condition, and if the manifold $\partial \Omega$ is $\mathcal{C}^{4}$, then the error is of order $3 / 2$. This fact is illustrated in section 6.2 with a constant density and a cylinder.

6. Cylindrical examples. In this section we provide a few canonical examples showing the contribution of the curvature effect on the solution.

Example 6.1 puts the problem of boundary source in the more general context of enforcing boundary conditions. Examples 6.2 and 6.3 describe, respectively, the cases of the spanwise and azimuthal components of the cylinder. A numerical application of kinematic boundary conditions is then provided in section 6.4.

6.1. Splitting the full heat equation. The method presented herein can be very useful when a scheme that does not control boundary conditions is used. Indeed, the problem

$$
\left\{\begin{array}{l}
\left.\frac{\partial \omega}{\partial t}-\nu \Delta \omega=g \text { in } \Omega \times\right] 0, T[ \\
\omega(x, 0)=\omega_{0}(x) \text { on } \Omega, \\
\left.\nu \mathcal{L}_{x} \omega(x, t)=F(x, t) \text { on } \partial \Omega \times\right] 0, T[
\end{array}\right.
$$

can be solved in the inner part of $\Omega$ by a numerical method not consistent on boundaries (or leading to a prohibitive computational cost when consistent), i.e., approximating the problem

$$
\left\{\begin{array}{l}
\left.\frac{\partial \omega}{\partial t}-\nu \Delta \omega=g \text { in } \Omega \times\right] 0, T[ \\
\omega(x, 0)=\omega_{0}(x) \text { on } \Omega
\end{array}\right.
$$

with arbitrary boundary conditions. Then one can measure the error on boundaries

$$
q(x, t)=\nu \mathcal{L}_{x} \omega(x, t)
$$

and use the present integral method to give explicitly (without the cost of another partial differential equation to solve) the solution of

$$
\left\{\begin{array}{l}
\left.\frac{\partial \omega}{\partial t}-\nu \Delta \omega=0 \text { in } \Omega \times\right] 0, T[ \\
\omega(x, 0)=0 \text { on } \Omega, \\
\left.\nu \mathcal{L}_{x} \omega(x, t)=F(x, t)-q(x, t) \text { on } \partial \Omega \times\right] 0, T[,
\end{array}\right.
$$

which is then approximated (at the appropriate order coming from Theorem 5.1 or Theorem 5.3) by

$$
\omega(x, t) \simeq \int_{0}^{t} \int_{\partial \Omega} \frac{-2 F(\xi, \tau)+2 q(\xi, \tau)}{1+2(\bar{\kappa}(\xi)-\beta(\xi)) \sqrt{\nu \tau / \pi}} \frac{e^{-(x-\xi)^{2} / 4 \nu(t-\tau)}}{(4 \pi \nu(t-\tau))^{3 / 2}} \mathrm{~d} \sigma(\xi) \mathrm{d} \tau,
$$


which can itself be computed by a time quadrature (for $t$ sufficiently small, typically for a time step when used in a numerical context) over a surface integral, using, for example, the midpoint rule:

$$
\omega(x, t) \simeq \int_{\partial \Omega} \frac{-2 t F(\xi, t / 2)+2 t q(\xi, t / 2)}{1+2(\bar{\kappa}(\xi)-\beta(\xi)) \sqrt{\nu t / 2 \pi}} \frac{e^{-(x-\xi)^{2} / 2 \nu t}}{(2 \pi \nu t)^{3 / 2}} \mathrm{~d} \sigma(\xi) .
$$

Note that the time quadrature based on an implicit Euler scheme is singular, while an explicit Euler scheme does not take curvature into account, despite the fact of being only first order.

Moreover, in order to provide more accuracy, this technique can be repeated on smaller intervals of time. The drawback in segmenting the time interval is that it reduces standard deviation of the Gaussian, which can possibly make the scheme underresolved, especially for three-dimensional computations. This approach, which couples the present integral method and a particle strength exchange (PSE) scheme (see [12]), has been successfully used for three-dimensional flow computations in [32], where boundary effects are the dominant effect.

Furthermore, one can also notice that (6.2)-(6.3) can be naturally parallelized if (6.2) is solved for homogeneous boundary conditions (i.e., $q=0$ ). Using this density estimation also provides a way to correct lack of regularity at the grid interface when performing domain decomposition at minimal cost.

6.2. The spanwise invariant cylinder. Let $\mathcal{B}_{2}(0, r)$ be the open ball of center 0 and radius $r$ in $\mathbb{R}^{2}$, let $\Omega=\mathbb{R}^{2} \backslash \mathcal{B}_{2}(0, r) \times \mathbb{R}$ be an infinitely long cylindrical body of $\mathbb{R}^{3}$, and let $\mathcal{C}=\partial \mathcal{B}_{2}(0, r)$.

One considers the heat equation and the spanwise component of its solution, which is related to a pure Neumann boundary condition. One then gets the spanwise heat layer of unity:

$$
\widetilde{\mathcal{H}}_{z}(x, t) 1=\nu \int_{0}^{t} \int_{\partial \Omega} n_{x} \cdot \nabla G_{\xi, \tau}(x, t) \mathrm{d} \sigma(\xi) \mathrm{d} \tau .
$$

Since the configuration is axisymmetric and spanwise invariant, one can set $x=$ $(r, 0,0)$ and $\xi=(r \cos \theta, r \sin \theta, 0)$ without loss of generality. By means of integration in the spanwise direction, one gets

$$
\begin{aligned}
\widetilde{\mathcal{H}}_{z}(x, t) 1 & =-\nu \int_{0}^{t} \int_{\mathcal{C}} \frac{1}{4 \pi \nu(t-\tau)} \frac{n_{x} \cdot(x-\xi)}{2 \nu(t-\tau)} \exp \left(-\frac{(x-\xi)^{2}}{4 \nu(t-\tau)}\right) \mathrm{d} \sigma(\xi) \mathrm{d} \tau \\
& =-\frac{1}{\pi} \int_{0}^{\nu t} \int_{-\pi}^{\pi} \frac{r}{8 u^{2}}(1-\cos \theta) \exp \left(-\frac{\left[(\cos \theta-1)^{2}+\sin ^{2} \theta\right] r^{2}}{4 u}\right) r \mathrm{~d} \theta \mathrm{d} u
\end{aligned}
$$

with $u=\nu(t-\tau)$. Noticing the symmetry around $\theta=0$, one gets by parity

$$
\widetilde{\mathcal{H}}_{z}(x, t) 1=-\frac{r^{2}}{2 \pi} \int_{0}^{\nu t} \int_{0}^{1} \frac{y}{u^{2}} \exp \left(-\frac{y r^{2}}{u}\right) \frac{1}{\sqrt{y(1-y)}} \mathrm{d} y \mathrm{~d} u
$$

with $y=(1-\cos \theta) / 2$, which integrates successively into

$$
\widetilde{\mathcal{H}}_{z}(x, t) 1=-\frac{1}{2 \pi} \int_{0}^{1} \frac{e^{-y r^{2} / \nu t}}{\sqrt{y(1-y)}} \mathrm{d} y=-\frac{1}{2} e^{-r^{2} / 2 \nu t} \mathrm{I}_{0}\left(\frac{r^{2}}{2 \nu t}\right),
$$

Copyright (c) by SIAM. Unauthorized reproduction of this article is prohibited. 


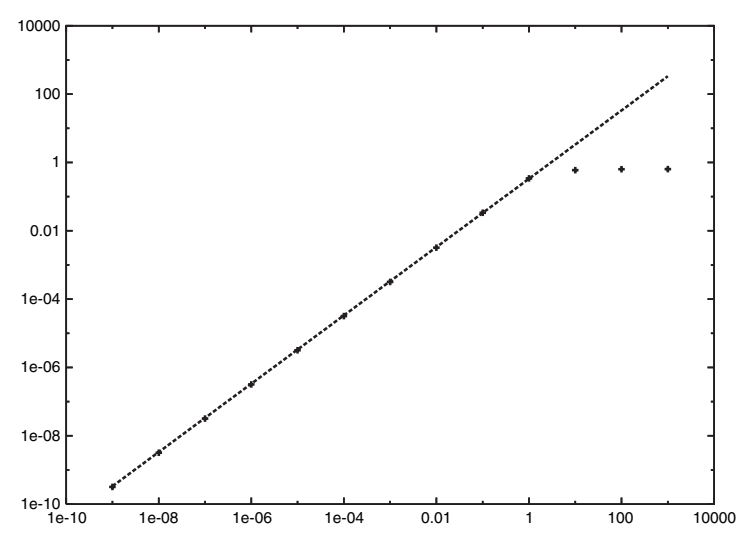

FIG. 5. Numerical values of heat layer $\widetilde{\mathcal{H}}_{z}(x, t) f_{x}$ with respect to time $t$ (dashed line represents the function $t / 3$ ).

where $\mathrm{I}_{0}$ is the modified Bessel function of first kind (i.e., the solution of $x^{2} y^{\prime \prime}+x y^{\prime}-$ $x^{2} y=0$ with $y(0)=1$ and $\left.y^{\prime}(0)=0\right)$. Since the mean curvature of $\partial \Omega$ is $\bar{\kappa}=1 / 2 r$, and noticing that

$$
\lim _{x \rightarrow+\infty} \sqrt{x} e^{-x} \mathrm{I}_{0}(x)=\frac{1}{\sqrt{2 \pi}},
$$

one finally has that $\widetilde{\mathcal{H}}_{z}(x, t) 1$ is equivalent to $-\bar{\kappa} \sqrt{\nu t / \pi}$ when $t$ tends to 0 , as expected by Lemma 4.2 and Theorem 5.1. One can notice that the present computation is performed on the exact surface $\partial \Omega$ instead of an approximation.

Moreover, in the present case, the density is smooth and the hypothesis of Theorem 5.3 holds; thus one can expect a full $3 / 2$ order of convergence. Indeed, a cylinder presents no torsion, and one can show that

$$
\lim _{x \rightarrow+\infty}\left(\sqrt{x} e^{-x} \mathrm{I}_{0}(x)-\frac{1}{\sqrt{2 \pi x}}\right) x^{3 / 2}=\frac{1}{8 \sqrt{2 \pi}},
$$

which proves the $3 / 2$ order of heat layer error on both the exact surface and its quadratic approximation. This example illustrate the statement of Theorem 5.3, i.e., that no torsion implies no error at first order in time for a constant source.

In order to exhibit the limit convergence order of Theorem 5.1, one has to choose an example for which Theorem 5.3 is not valid. Since the cylinder is torsion-free, one has to choose a density which is 1-Hölder continuous without being differentiable. The Euclidean norm satisfies this condition, and using the notation already set above, one considers the density

$$
f_{x}(\xi, \tau)=|x-\xi|=2 r \sqrt{y}
$$

thus evaluating the heat layer at point $x$ gives

$$
\widetilde{\mathcal{H}}_{z}(x, t) f_{x}=-\frac{r}{\pi} \int_{0}^{1} \frac{e^{-y r^{2} / \nu t}}{\sqrt{1-y}} \mathrm{~d} y .
$$

Integrating this integral symbolically is more difficult than the previous ones (though possible using erf functions). Figure 5 shows a certified 15-digit evaluation of the integral expression (6.6) with respect to time $t$. This actually exhibits a first order convergence since $f_{x}(x, t)=0$, and consequently $\widetilde{\mathcal{H}}_{z}(x, t) f_{x}=f_{x}(x, t)+\mathcal{O}(t)$. 
6.3. Vectorial kinematic boundary conditions and integral formulation of Chorin's algorithm in the cylinder case. In this section, one applies the present density estimation to Chorin's algorithm in the parabolic context (initially proposed in an hyperbolic context [7]), in the case of a circular cylinder.

Let $u: \Omega \times[0, T] \rightarrow \mathbb{R}^{3}$ be a velocity field satisfying the Stokes equations, and let $\omega$ be its associated vorticity field defined by $\omega=\operatorname{curl} u$ and satisfying the diffusion problem with kinematic boundary conditions:

$$
\left\{\begin{array}{l}
\left.\frac{\partial \omega}{\partial t}-\nu \Delta \omega=0 \text { in } \Omega \times\right] 0, T[ \\
u(x, t)=0 \text { on } \partial \Omega \times] 0, T[ \\
\omega(x, 0)=\omega_{0}(x) \text { on } \Omega
\end{array}\right.
$$

While computing $\omega$ from $u$ is obvious, building $u$ from $\omega$ is more difficult. In the full space $\Omega=\mathbb{R}^{3}$, one can use the three-dimensional Green kernel $G(x)=(4 \pi|x|)^{-1}$ through the Biot-Savart law

$$
u=\nabla G \hat{*} \omega=\int_{\mathbb{R}^{3}} \nabla G(\cdot-x) \wedge \omega(x) \mathrm{d} x .
$$

In the presence of boundaries, one can use symmetrizations of Biot-Savart laws around $\partial \Omega$ or more rigorously consider the single-layer integral formulation of (6.7) shown in [10]. Nevertheless, integral techniques are much less useful for this problem than the one presented herein, since the Green kernel and its gradient decrease much more slowly than Gaussian functions. A more competitive approach to computing the velocity from the vorticity is to introduce the Poisson equation on stream function with appropriate boundary conditions (see [30]) and eventually additional quantities such as potential stream to uncouple components of the three-dimensional Poisson equation (see [11]). In any case, it is possible to consider the operator $\mathcal{A}$, in the appropriate functional space, associating a velocity field $u=\mathcal{A} \omega$ to a vorticity field $\omega$, satisfying $\operatorname{curl} u=\omega$ and $\operatorname{div} u=0$ on $\Omega$ and $u \cdot n_{x}=0$ on $\partial \Omega$.

One considers the infinitely long circular cylinder $\Omega$ of axis $e_{z}$ and radius $R$, whose other tangential vector is denoted $e_{\theta}$ and whose normal vector is denoted $e_{r}$ (that is, the standard cylindrical coordinates).

Applying Chorin's method (whose convergence is proved in [8] in the case of the Stokes equation and its rotational formulation), this problem can be reduced to two parabolic problems with Neumann and Robin-Fourier (see [11]) boundary conditions. The first has homogeneous boundary conditions, which in cylindrical coordinates reads as

$$
\left\{\begin{array}{l}
\left.\frac{\partial \omega^{1}}{\partial t}-\nu \Delta \omega^{1}=0 \text { in } \Omega \times\right] 0, T[, \\
\omega^{1}(x, 0)=\omega_{0}(x) \text { on } \Omega, \\
\left.\nu \frac{\partial \omega_{z}^{1}}{\partial n}=0 \text { on } \partial \Omega \times\right] 0, T[, \\
\left.\nu \frac{\omega_{\theta}^{1}}{R}+\frac{\partial \omega_{\theta}^{1}}{\partial n}=0 \text { on } \partial \Omega \times\right] 0, T[, \\
\left.\omega_{r}^{1}=0 \text { on } \partial \Omega \times\right] 0, T[,
\end{array}\right.
$$

Copyright (c) by SIAM. Unauthorized reproduction of this article is prohibited. 
whose divergence-free and no-slip-through associated velocity field $u^{1}=\mathcal{A} \omega^{1}$ presents a priori nonzero tangential values ( $u^{1}$ is usually called spurious velocity). One then considers a second diffusion problem with zero initial condition,

$$
\left\{\begin{array}{l}
\left.\frac{\partial \omega^{2}}{\partial t}-\nu \Delta \omega^{2}=0 \text { in } \Omega \times\right] 0, T[, \\
\omega^{2}(x, 0)=0 \text { on } \Omega, \\
\left.\nu \frac{\partial \omega_{z}^{2}}{\partial n}=-\frac{\partial u_{\theta}}{\partial t} \text { on } \partial \Omega \times\right] 0, T[, \\
\left.\nu \frac{\omega_{\theta}^{2}}{R}+\frac{\partial \omega_{\theta}^{2}}{\partial n}=\frac{\partial u_{z}}{\partial t} \text { on } \partial \Omega \times\right] 0, T[, \\
\left.\omega_{r}^{2}=0 \text { on } \partial \Omega \times\right] 0, T[,
\end{array}\right.
$$

which allows us to link asymptotically (see [8]) the solution of (6.7) to the solutions of (6.9)-(6.10):

$$
\omega^{1}(t)+\omega^{2}(t)=\omega(t)+\mathcal{O}(t) .
$$

One can immediately notice that problem (6.10) is a heat equation with a zero initial condition and a vectorial Robin-Fourier boundary condition, on which one can apply component by component the density estimation presented herein, as long as boundary conditions are expressed in a basis in which the fundamental solution of heat equation is still Gaussian. One can thus introduce the matrix

$$
K=\left[\begin{array}{lll}
1 & 0 & 0 \\
0 & 1 & 0 \\
0 & 0 & 0
\end{array}\right], N=\left[\begin{array}{lll}
0 & 0 & 0 \\
0 & 1 & 0 \\
0 & 0 & 1
\end{array}\right], \text { and } \Gamma_{\theta}=\left[\begin{array}{ccc}
\cos \theta & \sin \theta & 0 \\
-\sin \theta & \cos \theta & 0 \\
0 & 0 & 1
\end{array}\right]
$$

so that in the canonical basis $\omega=\left(\omega_{x}, \omega_{y}, \omega_{z}\right)$, the boundary operator of equation (6.10) reads as

$$
\mathcal{L}_{x} \omega=\Gamma_{-\theta} K \Gamma_{\theta} \frac{\omega}{R}+\Gamma_{-\theta} N \Gamma_{\theta} \frac{\partial \omega}{\partial n}
$$

when expressed at point $x=(R \cos \theta, R \sin \theta, z)$. This allows us to write the boundary conditions of (6.10) under the form

$$
\nu \mathcal{L}_{x} \omega=\frac{\partial u}{\partial t} \wedge n_{x}
$$

One can use Theorem 5.1 and get by linearity

$$
\widetilde{\mathcal{H}}(x, t) f \simeq \Gamma_{-\theta}\left(K-\frac{N}{2}\right) \Gamma_{\theta} \frac{f(x, t)}{R}
$$

because the mean curvature of the cylinder in $\kappa=1 / 2 R$. Since the density of the potential giving $\omega$ satisfies the integral equation $\widetilde{\mu}-2 \widetilde{\mathcal{H}} \widetilde{\mu}=-2 \partial_{t} u \wedge n$, one gets

$$
\widetilde{\mu}(x, t) \simeq-2 \Gamma_{-\theta}\left(\operatorname{Id}-\frac{\sqrt{\nu t / \pi}}{R}(2 K-N)\right)^{-1} \Gamma_{\theta} \frac{\partial u}{\partial t} \wedge n_{x}
$$

Copyright $@$ by SIAM. Unauthorized reproduction of this article is prohibited. 
at the appropriate order, depending only on regularity of $u$ since the cylinder is a $\mathcal{C}^{\infty}$ differentiable manifold.

Setting $\varepsilon=\sqrt{\nu t / \pi} / R,(6.16)$ reads as

$$
\widetilde{\mu}(x, t) \simeq-2 \Gamma_{-\theta} P^{-1} \Gamma_{\theta} \frac{\partial u}{\partial t} \wedge n_{x}, \quad \text { where } \quad P=\left[\begin{array}{ccc}
1-2 \varepsilon & 0 & 0 \\
0 & 1-\varepsilon & 0 \\
0 & 0 & 1+\varepsilon
\end{array}\right] .
$$

This matrix $P$ is obvious to inverse, as long as $t$ is sufficiently small. One can notice that curvature effects are of different signs for $\omega_{\theta}$ and $\omega_{z}$ due to the Dirichlet part in the azimuthal direction.

Note also that if one considers only the $z$ direction (for a two-dimensional problem), the density estimation (6.17) can be written as

$$
\widetilde{\mu}_{z}(x, t) \simeq \frac{+2}{1+\frac{1}{R}(\sqrt{\nu t / \pi})} \frac{\partial u_{\theta}}{\partial t}(x, t) .
$$

6.4. Numerical example of kinematic boundary conditions. One considers the diffusion problem with time-periodic unknown $\omega: \Omega \times] 0,2 \pi] \mapsto \mathbb{R}^{3}$, still defined in a cylindrical domain $\Omega$, and kinematic boundary conditions:

$$
\left\{\begin{array}{l}
\left.\left.\frac{\partial \omega}{\partial t}-\nu \Delta \omega=0 \text { in } \Omega \times\right] 0,2 \pi\right] \\
\left.\left.u(x, t)=\cos t e_{\theta}(x) \text { on } \partial \Omega \times\right] 0,2 \pi\right]
\end{array}\right.
$$

where the velocity field $u(x, t)$ is built from $\omega$ by $u=\mathcal{A} \omega$, where operator $\mathcal{A}$ is based on formula (6.8), or by using a hybrid technique (see [11]).

Note that this problem is slightly more general than the one in the last section, because one has nonhomogeneous kinematic boundary conditions, whose main implication is that spurious velocity vanishes toward boundary value. Indeed, it has been shown (see [24]) that the parabolic problem

$$
\left\{\begin{array}{l}
\left.\left.\frac{\partial \omega}{\partial t}-\nu \Delta \omega=0 \text { in } \Omega \times\right] 0,2 \pi\right] \\
\left.\left.\nu \frac{\partial \omega_{z}}{\partial n}(x, t)=-\frac{\partial u_{\theta}}{\partial t}(x, t)=\sin t \text { on } \partial \Omega \times\right] 0,2 \pi\right] \\
\left.\left.\nu \frac{\partial \omega_{\theta}}{\partial n}(x, t)=\frac{\partial u_{z}}{\partial t}(x, t)=0 \text { on } \partial \Omega \times\right] 0,2 \pi\right] \\
\left.\left.\omega_{r}=0 \text { on } \partial \Omega \times\right] 0,2 \pi\right]
\end{array}\right.
$$

approximates well problem (6.19) in this particular context (i.e., when residual velocities coming from (6.20) with homogeneous conditions vanish toward $\partial_{t} u$ as stated above). One can notice that the solution is then invariant in $z$ and $\theta$.

Then let the functions

$$
\left\{\begin{aligned}
K^{+}(r) & =\mathfrak{R e}\left(K e_{0}(c r)\right)+\mathfrak{I m}\left(K e_{0}(c r)\right), \\
K^{-}(r) & =\mathfrak{R e}\left(K e_{0}(c r)\right)-\mathfrak{I m}\left(K e_{0}(c r)\right)
\end{aligned}\right.
$$

be based on Kelvin functions (sometimes also called Thompson functions), with $c=$ $1 / \sqrt{\nu}$. The function

$$
\omega^{*}(r, t)=\left(\alpha K^{-}(r)-\beta K^{+}(r)\right) \cos t-\left(\alpha K^{+}(r)+\beta K^{-}(r)\right) \sin t
$$


is then a time-periodic solution of $\partial_{t} \omega-\nu \Delta \omega=0$. Setting $A=c \sqrt{2} / 2\left|K e_{1}(c)\right|$ helps to check that $\beta-i \alpha=K e_{1}(c) / A$ is the only pair of parameters that makes $\omega^{*} e_{z}$ satisfy (6.20).

Let us consider (6.20) over a time step $] t_{n}, t_{n+1}\left[\right.$ with $t_{n}=n \delta t$ and its initial value denoted $\omega^{n}(x)$. The initial value for $n=0$ is set with the exact solution $\omega_{z}^{0}(x)=\omega^{*}\left(|x|, t_{n}\right)$ and other components of $\omega^{0}$ set to zero.

Playing with linearity of the heat equation (without approximation), (6.20) can be solved in two steps over ] $t_{n}, t_{n+1}$ [. The first step is the computation of the solution with arbitrary boundary conditions, in practice using a second order PSE scheme on a grid with a uniform cylindrical lattice (see [11]). The second step is the enforcement of the boundary condition, in the spirit of section 6.1.

Nevertheless, one can notice that truncated PSE schemes are consistent with a flux and can be tuned to provide homogeneous Neumann boundary conditions. The flux error denoted $q$ in section 6.1 can consequently be set to 0 in equations (6.3)-(6.5). The two steps can be naturally parallelized, and the second step can be computed with the integral scheme presented herein in the pure Neumann boundary condition context, which, using formula (6.18), reads as

$$
\omega_{z}\left(x, t_{n}+\delta t\right) \simeq \omega_{z}\left(x, t_{n}\right)-2 \int_{0}^{\delta t} \int_{\partial \Omega} \frac{G_{\xi, \tau}(x, \delta t)}{1+\frac{1}{R}(\sqrt{\nu \tau / \pi})} \sin \left(t_{n}+\tau\right) \mathrm{d} \sigma(\xi) \mathrm{d} \tau,
$$

where $R$ is the cylinder radius, without any action on other components since 0 is solution. Note that Theorem 5.3 is valid only at $t_{n}=0$; thus this density evaluation is first order (from Corollary 5.2). One can then use a time quadrature to compute $\omega\left(x, t_{n+1}\right)$, such as using the midpoint rule (see (6.5) with $q=0$ ).

This one-dimensional reducible example allows us to compare the three-dimensional algorithm presented herein with the two-dimensional algorithm from [24] given for Neumann boundary conditions, and compare both of them with the exact solution. These three quantities are plotted in Figure 6 at times $t=0.75,1,1.5$, and 2 . The curves show a good agreement qualitatively, but the main result is that the algorithm allows us to enforce very well the kinematic boundary conditions: the residual velocity (rebuilt from vorticity $\omega$ by the operator $\mathcal{A}$ defined in section 6.3) is close to $10^{-6}$, and this code was run in simple precision.

7. Toroidal examples. This section aims to illustrate the optimality of the convergence ratio obtained by Theorem 5.1. In order to proceed, one has to consider an example for which Theorem 5.3 is not valid.

The differences in the hypotheses of Theorems 5.1 and 5.3 lead us to consider either a nondifferentiable 1-Hölder continuous function on a torsion-free surface (the case already studied in section 6.2), or a smooth function density defined on a surface presenting torsion (i.e., whose tensor of map third derivatives is not identically zero).

In order to build such a surface and analyze properties of the integrodifferential operator $\widetilde{\mathcal{H}}$ on it, one considers the heat equation with pure Neumann boundary conditions:

$$
\left\{\begin{array}{l}
\left.\left.\frac{\partial \omega}{\partial t}-\nu \Delta \omega=0 \text { in } \Omega \times\right] 0, \delta t\right], \\
\left.\left.\nu \frac{\partial \omega}{\partial n}(x, t)=F(x, t) \text { on } \partial \Omega \times\right] 0, \delta t\right], \\
\omega(x, 0)=0 \text { on } \partial \Omega,
\end{array}\right.
$$

Copyright $@$ by SIAM. Unauthorized reproduction of this article is prohibited. 

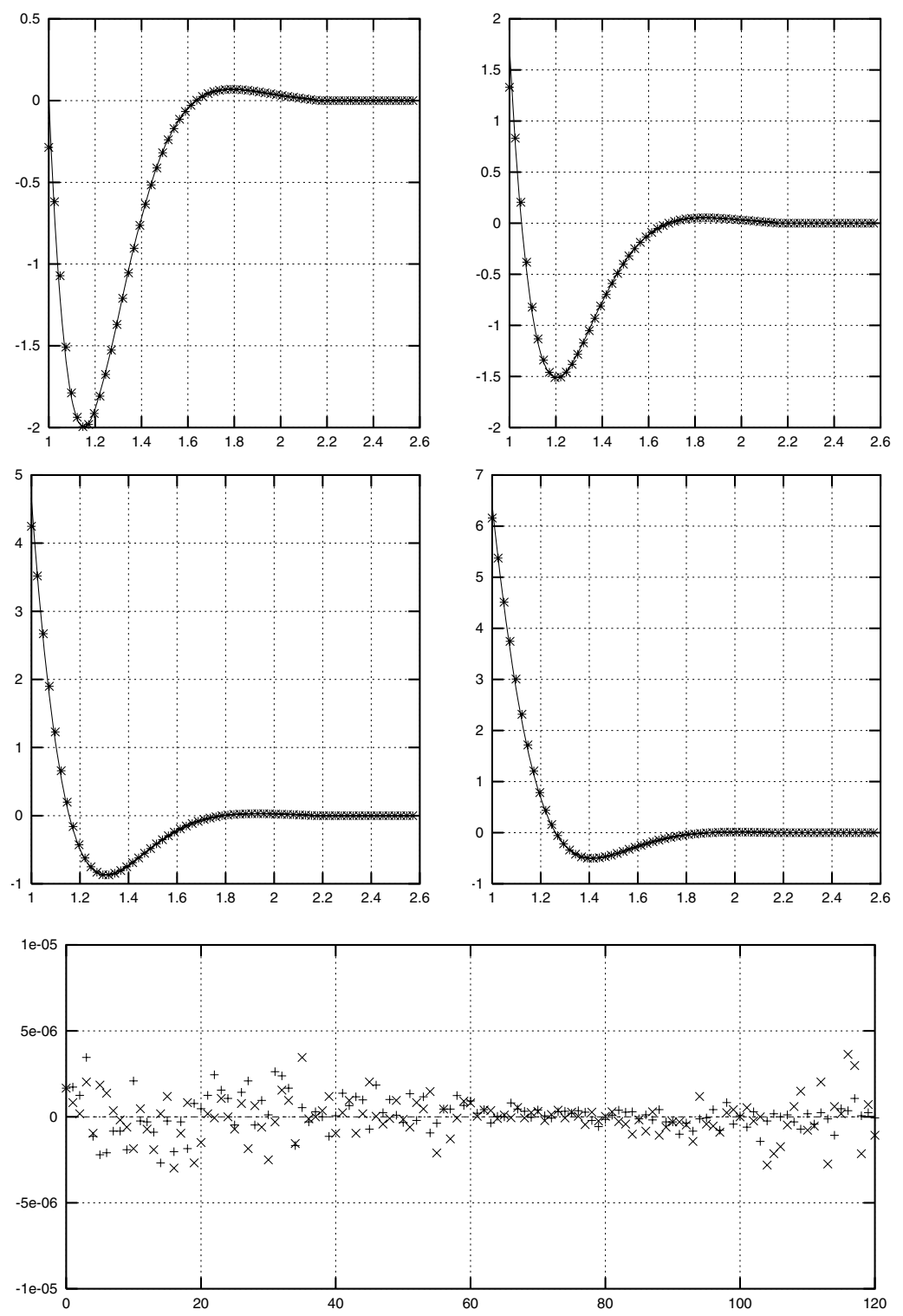

FIG. 6. Numerical solutions compared to the exact axisymmetric and time-periodic solution of the heat equation at times $t=0.75,1.0,1.5,2.0$, with exact solution (-), three-dimensional scheme $(+)$, and two-dimensional scheme $(\times)$ from [24]. Bottom: residual tangential velocity versus time step number $n$ (time step $\delta t=0.025$ ).

where $\partial \Omega$ is successively the usual torus in section 7.1 and then modified with harmonic perturbations introducing torsion in sections 7.2 and 7.3. Large and small wavelength perturbations are involved, generating, respectively, a "twisted" and a "rippled" torus.

Instead of solving the heat equation, we will discuss properties of the related operator $\widetilde{\mathcal{H}}$ defined by formula $(2.2)$, which reads as follows:

$$
\widetilde{\mathcal{H}}(x, t) 1=-\frac{\nu}{16 \pi^{3 / 2}} \int_{0}^{t} \int_{\partial \Omega} \frac{(x-\xi) \cdot n_{x}}{(\nu(t-\tau))^{5 / 2}} e^{-|x-\xi|^{2} / 4 \nu(t-\tau)} \mathrm{d} \sigma(\xi) \mathrm{d} \tau .
$$

Copyright (c) by SIAM. Unauthorized reproduction of this article is prohibited. 

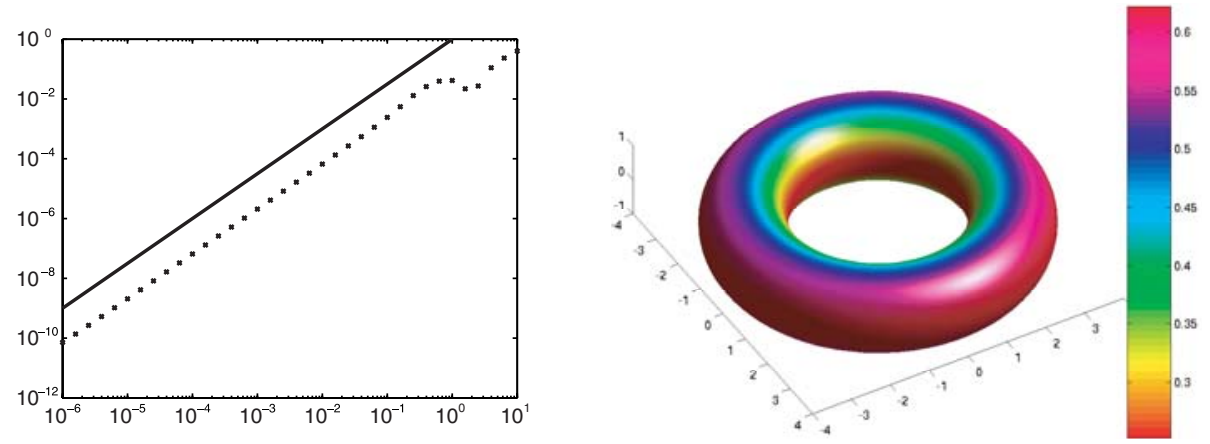

FIG. 7. Right: plot of this surface, with color being the curvature coefficient given by formula (7.12). Left: pointwise error of the estimate of $\widetilde{\mathcal{H}}(x, t) 1$ in $\theta_{0}=\pi / 2$ versus $\nu t$ ( $\times$ is formula (7.13), and the solid line is $\left.(\nu t)^{3 / 2}\right)$.

7.1. The torsionless torus. One sets two radii $r$ and $R>r$, and two angles $\theta \in[-\pi, \pi[$ and $\zeta \in[-\pi, \pi[$, both defined modulo $2 \pi$. The torus $\mathbb{T}$ is then the image of these domains by the function

$$
f(\theta, \zeta)=((R+r \cos \theta) \cos \zeta,(R+r \cos \theta) \sin \zeta, r \sin \theta),
$$

which satisfies

$$
\left\|\frac{\partial f}{\partial \theta} \wedge \frac{\partial f}{\partial \zeta}\right\|_{2}=r I_{\theta} \quad \text { with } \quad I_{\theta}=(R+r \cos \theta)>0 .
$$

The resulting surface is shown in Figure 7.

One can consider the points on the $\zeta=0$ section, defined by

$$
x=f\left(\theta_{0}, 0\right)=\left(I_{\theta_{0}}, 0, r \sin \theta_{0}\right),
$$

as arbitrary points of the surface without loss of generality, since the torus is globally $\zeta$-invariant. The normal vector to $\mathbb{T}$ in $x$ is then

$$
n_{x}=\left(\cos \theta_{0}, 0, \sin \theta_{0}\right) .
$$

Moreover, in order to describe a neighborhood of $x$, one also defines

$$
\xi=f(\theta, \zeta)=\left(I_{\theta} \cos \zeta, I_{\theta} \sin \zeta, r \sin \theta\right) .
$$

The integrodifferential operator defined by formula (7.2) reads as

$$
\widetilde{\mathcal{H}}(x, t) 1=-\frac{1}{2 \pi^{3 / 2}} \int_{0}^{4 \nu t} \int_{\partial \Omega} \frac{(x-\xi) \cdot n_{x}}{u^{5 / 2}} e^{-|x-\xi|^{2} / u} \mathrm{~d} \sigma(\xi) \mathrm{d} u
$$

for $u=4 \nu(t-\tau)$. In order to obtain a two-dimensional integral calculus, one can set

$$
U_{t}(\theta, \zeta)=\frac{(x-\xi(\theta, \zeta))^{2}}{4 \nu t}
$$

so that

$$
\int_{0}^{4 \nu t} e^{-(x-\xi(\theta, \zeta))^{2} / u} u^{-5 / 2} \mathrm{~d} u=\frac{\mathrm{E}\left(U_{t}(\theta, \zeta)^{1 / 2}\right)-U_{t}(\theta, \zeta)^{1 / 2} e^{-U_{t}(\theta, \zeta)}}{U_{t}(\theta, \zeta)^{3 / 2}}(4 \nu t)^{3 / 2},
$$



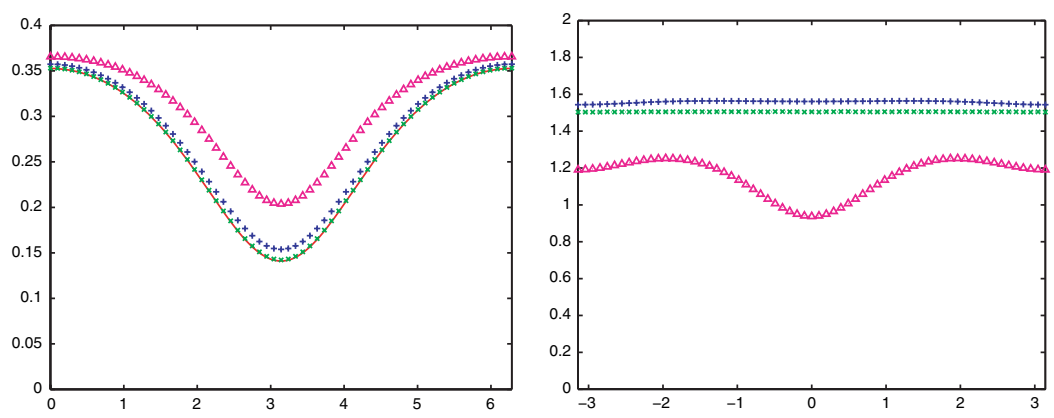

FIG. 8. Convergence of estimates for the torsionless torus. Left: uniform convergence of $(\nu \delta t)^{-1 / 2} \widetilde{\mathcal{H}}(x, \delta t) 1$ toward $\bar{\kappa}(x) / \sqrt{\pi}$ versus angle $\theta_{0}$. Right: resulting numerical order $N\left(\nu \delta t, \theta_{0}\right)$ obtained by formula (7.14) versus angle $\theta_{0}$. Legend: $\triangle$ is $\nu \delta t=1,+i s \nu \delta t=0.1$, and $\times$ is $\nu \delta t=0.01$.

where $\mathrm{E}$ is the scaled erf complementary function defined by

$$
\mathrm{E}(x)=-\int_{x}^{+\infty} e^{-z^{2}} \mathrm{~d} z=\frac{\sqrt{\pi}}{2}(\operatorname{erf}(x)-1) .
$$

This leads to a new expression for $\widetilde{\mathcal{H}}(x, t) 1$ :

$$
-\frac{r}{(4 \pi \nu t)^{3 / 2}} \int_{0}^{\pi} \int_{\theta_{0}-\pi}^{\theta_{0}+\pi} \frac{\mathrm{E}\left(U_{t}(\theta, \zeta)^{1 / 2}\right)-U_{t}(\theta, \zeta)^{1 / 2} e^{-U_{t}(\theta, \zeta)}}{U_{t}(\theta, \zeta)^{3 / 2}}(x-\xi(\theta, \zeta)) \cdot n_{x} I_{\theta} \mathrm{d} \theta \mathrm{d} \zeta .
$$

This two-dimensional integral is then computed by a fifth order Gauss-Legendre quadrature formula with $2000^{3}$ elements, once the singularity in $\left(\theta_{0}, 0\right)$ has been smoothed by setting $\theta=\theta_{0} \pm \hat{\theta}^{s}$ and $\zeta=\hat{\zeta}^{s}$ with $s=3$.

It can be shown (but is not developed herein) that the mean curvature in $x=$ $f(\theta, \zeta)$ is given by

$$
\bar{\kappa}(x)=\frac{1}{2}\left(\frac{1}{r}+\frac{\cos \theta}{R+r \cos \theta}\right) .
$$

Theorem 5.3 then predicts, since the torus is a $\mathcal{C}^{\infty}$ torsionless two-dimensional submanifold of $\mathbb{R}^{3}$, that

$$
\widetilde{\mathcal{H}}(x, t) 1-\bar{\kappa}(x) \sqrt{\frac{\nu t}{\pi}}=\mathcal{O}\left(t^{3 / 2}\right) .
$$

One verifies that this $3 / 2$ order is reached with the computation of the difference of the two expressions above for $x=\pi / 2$. The left-hand picture in Figure 7 shows that indeed the difference scales as $t^{3 / 2}$. In order to measure the convergence more uniformly, one introduces the numerical order of convergence $N(t)$ defined by

$$
N\left(\nu \delta t, \theta_{0}\right)=\log _{10}\left(\frac{\widetilde{\mathcal{H}}(x, \delta t) 1-\bar{\kappa}(x) \sqrt{\frac{\nu \delta t}{\pi}}}{\widetilde{\mathcal{H}}(x, \delta t / 10) 1-\bar{\kappa}(x) \sqrt{\frac{\nu \delta t / 10}{\pi}}}\right)
$$

with $x=f\left(\theta_{0}, 0\right)$ chosen on the $\zeta=0$ section (which is the generality since this torus is $\zeta$-invariant). This function is plotted in the right-hand graph of Figure 8 and shows a convergence toward the $3 / 2$ order everywhere. The convergence order suggested by Theorem 5.3 is consequently optimal. 

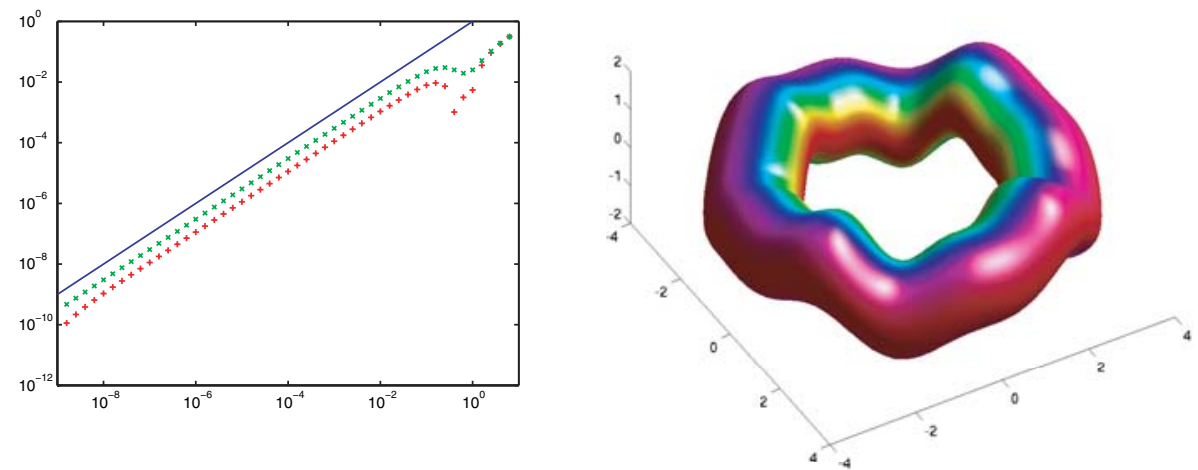

FIG. 9. Right: plot of the twisted torus (color is the same as for Figure 7). Left: pointwise error of the estimate of $\widetilde{\mathcal{H}}(x, t) 1$ versus $\nu t$ ( $\times$ is formula $(7.13)$ for $\theta_{0}=\pi / 4,+$ is formula $(7.13)$ for $\theta_{0}=\pi / 2$, and the solid line is $\left.\nu t\right)$.
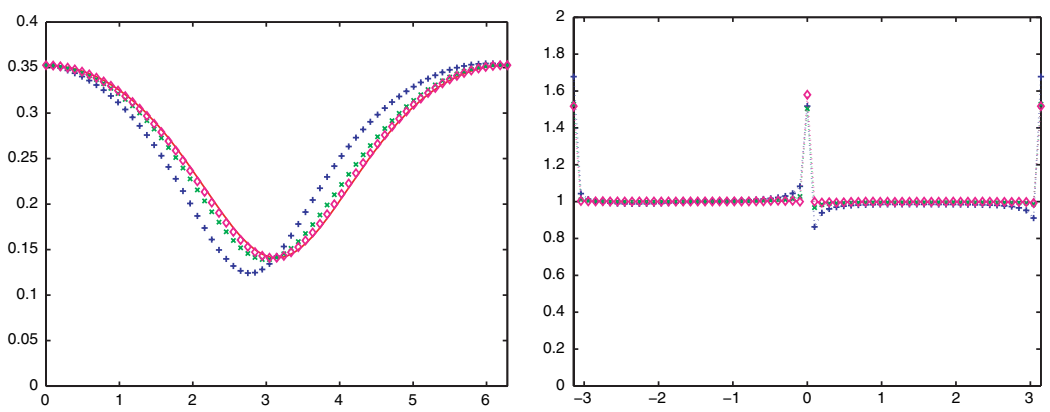

FIG. 10. Convergence of estimate for the twisted torus. Left: uniform convergence of $(\nu \delta t)^{-1 / 2} \widetilde{\mathcal{H}}(x, \delta t) 1$ toward $\bar{\kappa}(x) / \sqrt{\pi}$ versus angle $\theta_{0}$. Right: resulting numerical order $N\left(\nu \delta t, \theta_{0}\right)$ obtained by formula (7.14) versus angle $\theta_{0}$. Legend: + is $\nu \delta t=10^{-2}, \times$ is $\nu \delta t=10^{-3}$, and $\triangle$ is $\nu \delta t=10^{-4}$.

7.2. The "twisted" torus. In order to introduce torsion effects to the geometry, one considers two strictly positive numbers $\bar{A}$ and $\bar{m}$ and the function

$$
g(\zeta)=\bar{A}(2 \sin (\bar{m} \zeta)-\sin (2 \bar{m} \zeta)),
$$

which satisfies $g(0)=g^{\prime}(0)=g^{\prime \prime}(0)=0$ and $g^{\prime \prime \prime}(0)=6 \bar{A} \bar{m}^{3} \neq 0$.

The surface defined by

$$
f(\theta, \zeta)+g(\zeta) e_{z}
$$

with $\bar{A}=0.2$ and $\bar{m}=4$ (where $e_{z}$ denotes the third vector of the canonical basis of $\mathbb{R}^{3}$ ) is called herein the "twisted" torus and is plotted in Figures 9 and 10.

It presents nonzero torsion everywhere on the section $\zeta=0$, except for $\theta_{0}=0$ and $\theta_{0}=\pi$ for which the mapping is tangential (thus introduces no torsion), without changing curvature, slope, and location of this section when compared to the torsionless torus discussed in the last section (note that in this case the Jacobian is not as obvious as before and is thus not explicitly given herein). 

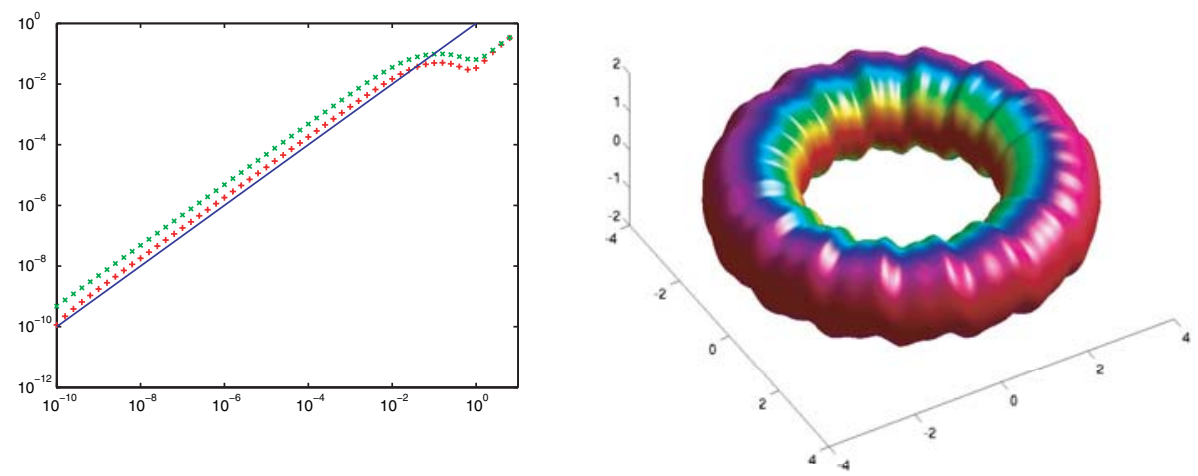

FIG. 11. Right: plot of the rippled torus (color is the same as for Figure 7). Left: pointwise error of the estimate of $\widetilde{\mathcal{H}}(x, t) 1$ versus $\nu t$ ( $\times$ is formula (7.13) for $\theta_{0}=\pi / 4,+$ is formula (7.13) for $\theta_{0}=\pi / 2$, and the solid line is $\left.\nu t\right)$.
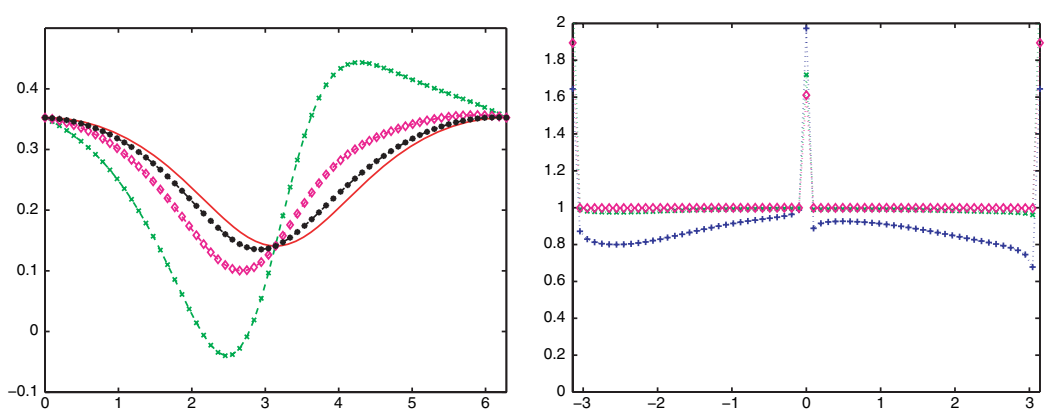

FIG. 12. Convergence of estimate for the rippled torus. Left: uniform convergence of $(\nu \delta t)^{-1 / 2} \widetilde{\mathcal{H}}(x, \delta t) 1$ toward $\bar{\kappa}(x) / \sqrt{\pi}$ versus angle $\theta_{0}$. Right: resulting numerical order $N\left(\nu \delta t, \theta_{0}\right)$ obtained by formula (7.14) versus angle $\theta_{0}$. Legend: + is $\nu \delta t=10^{-2}, \times$ is $\nu \delta t=10^{-3}, \triangle$ is $\nu \delta t=10^{-4}$, and $*$ is $\nu \delta t=10^{-5}$.

According to Theorem 5.1 and the strong need in Theorem 5.3 of the torsion-free surface to reach order $3 / 2$, one expects in the present case to observe a first order convergence rate, except for the singular value of $\theta_{0}$ mentioned above.

7.3. The "rippled" torus. In this section one considers the same kind of torus as in section 7.2 , with perturbation parameters chosen as $\bar{A}=0.05$ and $\bar{m}=16$. This example provides a torsion 16 times stronger than the previous one, which makes torsion effects even clearer. Indeed, one can see in Figure 11 that convergence is globally first order, as observed for the twisted torus, but with a shift of accuracy due to stronger torsion effects.

Figure 12 shows that the first order is induced by a broken symmetry led by the torsion tensor, but also that the order is still constant over the section, except for $\theta_{0}=0$ for which the torsion effect is tangential; thus it does not act on body torsion and allows a $3 / 2$ convergence order at this special point.

8. Conclusion. In this article, we have proved that the solution of the heat equation whose sources are provided only at boundaries can be explicited analytically up to order $3 / 2$, exhibiting a square root in time depending on the boundary curvature 
and the Dirichlet part of the Robin-Fourier coefficients of the boundary conditions. The solution is expressed in its integral formulation, involving a Gaussian kernel and a surface density. Most of the present study focuses on properties of this density.

The main result obtained herein is that since the density is analytically provided, one gets a very fast estimation of the solution of the heat equation for early times. This leads to a fast numerical scheme for kinematic boundary conditions or in addition to a scheme not satisfying algebraically the Robin-Fourier boundary condition.

The order depends on whether the manifold defining the domain boundary is torsionless or not, and on the manifold and the density regularities. Since small times are considered, the Gaussian kernel of the heat equation has small standard deviation, and thus its effect is localized (if not compactly supported). Therefore classical results have been extended to a class of noncompact manifolds, satisfying a few properties denoted (C1)-(C5).

We first discussed the error estimation due to restriction and then the error resulting from the substitution of the manifold by its best quadratic approximant. The error coming from the flattening process was also discussed, obtaining finally an integral expression which can be symbolically carried out.

Several applications illustrate that the limit convergence rates given by Theorems 5.1 and 5.3 are optimal. As examples, we investigate numerically the two- and three-dimensional cylinders, whose different eigenvectors of the curvature matrix induce density anisotropy. These cylindrical examples allow us to show the effect of density regularity on the double heat layer. The effect of manifold torsion is finally investigated for smoothly perturbated toroidal manifolds.

Acknowledgments. The author thanks Georges-Henri Cottet and Petros Koumoutsakos for their helpful contribution in the early work leading to the threedimensional results.

\section{REFERENCES}

[1] S. Alinhac and P. GÉrard, Opérateurs pseudo-différentiels et théorème de Nash-Moser, Editions du CNRS, Meudon, France, 1991.

[2] C. Anderson and C. Greengard, On vortex methods, SIAM J. Numer. Anal., 22 (1985), pp. $413-440$.

[3] J. T. BEALE, A convergent boundary integral method for three-dimensional water waves, Math. Comp., 70 (2000), pp. 977-1029.

[4] J. T. Beale, T. Y. Hou, and J. S. Lowengrub, Convergence of a boundary integral method for water waves, SIAM J. Numer. Anal., 33 (1996), pp. 1797-1843.

[5] J. Chazarin And A. Piriou, Introduction à la théorie des équations aux dérivées partielles linéaires, Gauthier-Villars, Paris, 1981.

[6] A. K. Chaniotis, D. Poulikakos, and P. Koumoutsakos, Remeshed smoothed particle hydrodynamics for the simulation of viscous and heat conducting flows, J. Comput. Phys., 182 (2002), pp. 67-90.

[7] A. J. Chorin, Numerical study of slightly viscous flow, J. Fluid Mech., 57 (1973), pp. 785-796.

[8] G.-H. Соттет, A vorticity creation algorithm for the Navier-Stokes equations in arbitrary domain, in Navier-Stokes Equations and Related Nonlinear Problems, A. Sequeira, ed., Plenum, New York, 1995.

[9] G.-H. Cottet and P. D. Koumoutsakos, Vortex Methods, Theory and Practice, Cambridge University Press, Cambridge, UK, 2000.

[10] G.-H. Cottet and P. Poncet, Particle methods for direct numerical simulations of threedimensional wakes, J. Turbul., 3 (2002), pp. 1-9.

[11] G.-H. Cотtet And P. Poncet, Advances in direct numerical simulations of $3 D$ wall-bounded flows by vortex-in-cell methods, J. Comput. Phys., 193 (2004), pp. 136-158.

[12] P. Degond And S. Mas-Gallic, The weighted particle method for convection-diffusion equations, Math. Comp., 53 (1989), pp. 485-526. 
[13] L. Dragos And A. Dinu, A direct boundary integral method for the three-dimensional lifting flow, Comput. Methods Appl. Mech. Engrg., 127 (1995), pp. 357-370.

[14] A. Friedman, Partial Differential Equations of Parabolic Type, Prentice-Hall, Englewood Cliffs, NJ, 1964.

[15] L. Greengard and V. Rokhlin, A fast algorithm for particle simulation, J. Comput. Phys., 73 (1987), pp. 325-348.

[16] Ph. Guillaume, A. Huard, and C. Le Calvez, A block constant approximate inverse for preconditioning large linear systems, SIAM J. Matrix Anal. Appl., 24 (2003), pp. 822-851.

[17] D. J. Haroldsen And D. Meiron, Numerical calculation of three-dimensional interfacial potential flows using the point vortex method, SIAM J. Sci. Comput., 20 (1998), pp. 648-683.

[18] J. L. HESs, Review of integral-equation techniques for solving potential flow problems with emphasis on the surface-source method, Comput. Methods Appl. Mech. Engrg., 5 (1975), pp. $145-196$.

[19] J. L. Hess, Panel methods in computational fluid dynamics, Ann. Rev. Fluid Mech., 22 (1990), pp. $255-274$.

[20] S. ITÔ, Fundamental solutions of parabolic differential equations and boundary value problems, Japan J. Math., 27 (1957), pp. 55-102.

[21] J. Katz and A. Plotkin, Low-Speed Aerodynamics, McGraw-Hill, New York, 1991.

[22] J. Kim And P. Moin, Application of a fractional-step method to incompressible Navier-Stokes equations, J. Comput. Phys., 59 (1985), pp. 308-323.

[23] R. B. Kinney and Z. M. Cielak, Analysis of unsteady viscous flow past an airfoil. Part I: Theoretical development, AIAA J., 15 (1977), pp. 1712-1717.

[24] P. D. Koumoutsakos, A. Leonard, and F. Pepin, Boundary conditions for viscous vortex methods, J. Comput. Phys., 113 (1994), p. 52.

[25] P. D. Koumoutsakos And A. Leonard, High-resolution simulations of the flow around an impulsively started cylinder using vortex methods, J. Fluid Mech., 296 (1995), pp. 1-38.

[26] S. V. Kozlov, I. K. Lifanov, and A. A. Mikhailov, A new approach to mathematical modelling of flow of ideal fluid around bodies, Soviet J. Numer. Anal. Math. Modelling, 6 (1991), pp. 209-222.

[27] E. E. LEvI, Sulle equazioni lineari totalmente ellittiche alle derivate parziali, Rend. Circ. Mat. Palermo, 24 (1907), pp. 275-317.

[28] O. A. Ladyzhenskaya, V. A. Solonnikov, and N. N. Ural'ceva, Linear and Quasilinear Equations of Parabolic Type, AMS, Providence, RI, 1968.

[29] M. J. Lighthill, Boundary Layer Theory, Oxford University Press, Oxford, UK, 1963.

[30] M. L. Ould-Sahili, G.-H. Cottet, and M. El Hamraoui, Blending finite-differencies and vortex methods for incompressible flow computations, SIAM J. Sci. Comput., 22 (2000), pp. 1655-1674.

[31] W. Pogorzelski, Propriétés des intégrales de l'équation parabolique normale, Ann. Polon. Math., 4 (1957), pp. 61-92.

[32] P. Poncet, Topological aspects of the three-dimensional wake behind rotary oscillating circular cylinders, J. Fluid Mech., 517 (2004), pp. 27-53.

[33] P. Ramachandran, S. C. Rajan, and M. Ramakrishna, A fast, two-dimensional panel method, SIAM J. Sci. Comput., 24 (2003), pp. 1864-1878.

[34] A. RATHSFELD, The invertibility of the double layer potential operator in the space of continuous functions defined on a polyhedron: The panel method, Appl. Anal., 45 (1992), pp. $135-177$.

[35] J. E. Romate, Local error analysis of three-dimensional panel methods in terms of curvilinear surface coordinates, SIAM J. Numer. Anal., 27 (1990), pp. 529-542.

[36] M. TAYlor, Pseudodifferential Operators, Princeton University Press, Princeton, NJ, 1981.

[37] J. C. WU, Numerical boundary conditions for viscous flow problems, AIAA J., 14 (1976), pp. 1042-1049.

Copyright (C) by SIAM. Unauthorized reproduction of this article is prohibited. 